Accepted version of the paper published in Journal of Sound and Vibration, 484:115523, 2020, https://doi.org/10.1016/j.jsv.2020.115523.

Corresponding author: altay@lbb.rwth-aachen.de

\title{
Mathematical modeling and optimization scheme for omnidirectional tuned liquid column dampers
}

\author{
Behnam Mehrkian, Okyay Altay
}

RWTH Aachen University, Department of Civil Engineering, Mies-van-der-Rohe-Str. 1, Aachen 52074, Germany

\begin{abstract}
As a well-known and reliable control device, tuned liquid column dampers (TLCDs) have been investigated numerically and experimentally and implemented in a number of structures over the last three decades. However, TLCDs basically suffer from the lack of multidirectionality, which is the critical need for real structures, in particular under random vibrations such as wind and earthquake excitations. This aspect has garnered the attention of the structural control community to modify this promising damper to achieve more efficiency and to extend its application range to multidirectional vibrations. This paper proposes a mathematical modeling and optimization approach for omnidirectional tuned liquid column dampers (O-TLCDs). As an improved and reformed TLCD, O-TLCDs are formed by circularly distributed of $n$ (integer $n \geq 3$ ) L-arms about a common joint point at the center, through which all L-arms are connected to each other. Thanks to this layout, O-TLCDs can control structures with full counteracting force capacity in all transversal directions regardless of the excitation angle of incidence. This paper, in the first step, proposes the governing equation of motion of O-TLCDs, for which Lagrange's principle is employed, and the equation of motion of the coupled O-TLCD-structure system. In doing so, a formal solution to determine the degree of freedom (DoF) of the O-TLCD is introduced, which proves independence of the O-TLCD response from the number of L-arms as well as from the angle of excitations. Second, for designing O-TLCDs, a set of design criteria and a general optimization scheme, which accommodate the online simulation of coupled O-TLCD-structure system under arbitrary excitations, are proposed. Consequently, without adding extra complication coming from extra DoFs to the motion equation of the damper, the O-TLCD functions as an enhanced liquid damper for multidirectional vibration attenuation. Next, using the O-TLCDs designed with different mass ratios, numerical simulations of O-TLCD-structure systems are conducted under seismic loads, free vibration, harmonic excitation and white noise and the controlled and uncontrolled responses of the systems are assessed in the time and the frequency domain. Here, the role of important parameters such as the mass ratio, the head loss coefficient, the liquid deflection and the excitation amplitude are evaluated and the influence of varying conditions on the efficiency of the O-TLCD are discussed. Results demonstrate that the proposed O-TLCD can be well tuned to the structure and markedly control the peak and the RMS of responses of the structure. In the
\end{abstract}


end, an experimental study on a prototype O-TLCD is performed using a shaking table, which verifies the proposed mathematical modeling approach.

Keywords: TLCD, O-TLCD, structural control, passive vibration control, omnidirectional

\section{Introduction}

Vibratory systems such as structures built by conventional methodologies, which may everyday end up with more massive and stiffer structural elements, suffer from inherent low damping to face with stochastic and dynamic excitations. Structural control field, on the other hand, has shed light on dealing with dynamic vibrations compatibly, which was founded by the pioneering work of Yao [1] and profoundly reviewed (e.g. see [2, 3]). Structural control applications can be classified as passive, semi-active, active, and hybrid control systems [4-7].

Due to their simple concept and reliability, the widespread applications of tuned dampers, such as tuned mass dampers (TMDs) and tuned liquid dampers (TLDs), which can be accommodated in one or more of the mentioned classes have been noticeable. As a more matured tuned damper, which eliminates the need for problematic installations of TMDs and rectified the substantial inactive liquid of TLDs, the tuned liquid column damper (TLCD) [8, 9] is invented. A number of notable studies on TLCD may include the mathematical description of TLCDs and their optimum parameters using sinusoidal and/or random vibrations, such as [10 19, passive TLCDs, such as [20 31], and semi-active and active TLCDs, such as [32 41].

In parallel, the attention of the structural control community has been garnered to study the mathematical description of TLCDs operating in other degrees of freedom (DoFs) than the unidirectional translational movement. Hochrainer et al. [42] presented a torsional TLCD with ring-shaped geometry for controlling torsional vibrations of structures; in addition, the paper employed two other TLCDs for the horizontal translational DoFs and presented also experimental results. Similar ring-shaped geometric layout was numerically investigated for plan-asymmetric high-rise buildings exposed to an earthquake with prescribed angle of excitation [43], for simulation of a 27-story building with eccentricity under wind load [44] and for attenuation of the edgewise vibrations of wind turbine blades [45]. These studies showed that annular TLCDs can be effective when exposed to torsional response.

To control multiple degrees of freedom, Heo et al. [46] proposed a tuned liquid mass damper (TLMD), which was basically a TLCD mounted on natural rubbers, to reduce the translational responses in the weak and strong directions of a building. The TLMD worked as a TLCD in one direction where the liquid movement was employed and as a TMD in the orthogonal direction where the total weight of the TLCD was merely the tuned mass. Experimental results showed the TLMD could be tuned to two frequencies for bidirectional response reduction. Min et al. [47] proposed a two-way liquid damper, which was a TLCD with wider dimension perpendicular to the TLCD's plane, so that the damper behaved like a TLCD in one direction and a TLD in the orthogonal one; the efficiency of the damper was verified for a 64-story building under wind. Similar two-way TLCD was applied by Lee et al. [48] where the inclination of the excitation angle ranging from 0 to $90^{\circ}$ was also studied.

Email addresses: mehrkian@lbb.rwth-aachen.de (Behnam Mehrkian), altay@lbb.rwth-aachen.de (Okyay 
Further multidirectional TLCDs were introduced using plus-shaped by Sakai and Takaeda [49], crossed tube-like by Zhang et al. [50], and rectangular-based by Hitchcock et al. [51] layouts. A bidirectional TLCD, which consisted of four vertical columns connected with four horizontal ones, was proposed by Rozas et al. [52. The damper required less liquid than two independent TLCDs and acted as a TLCD in two orthogonal directions. Shaking-table tests with 45-degree orientation identified two distinct natural frequencies of the damper. Numerical investigations of a 3-story scaled model under seismic loads showed that the proposed bidirectional TLCD can control the roof displacements more than the roof accelerations in two first vibrational modes. Adopting this bidirectional TLCD, Tong et al. [53] studied controlling the pitch and the roll motions of wind turbines in the fore-aft and side-to-side directions, which brought reduction in damage loads of the tower and the fluctuations of the rotor speed and generator power. Based on the concept of conventional TLCDs, Coudurier et al. [54] introduced a tuned liquid multi-column damper in three different configurations to control simultaneously the pitch and roll motions of floating wind turbine platforms. The liquid dampers were composed of $N$ vertical columns, which were connected either simply by horizontal columns over each other or by interconnected horizontal columns. Depending on the arrangement of the horizontal columns, at least $N-1$ DoFs were used to formulate the equations of motion of the dampers. They concluded that, in comparison with crosswise layout of multiple TLCDs over each other, the interconnected-column arrangement can result in a better performance due to its robustness against wave incidence and less parasitic-oscillation creation.

Due to changing excitation direction, translational vibrations, such as the one induced by winds and earthquakes on high-rise structures, arise a challenge for structural control. Based on previously proposed TLCD configurations, a star shaped TLCD with N vertical columns seems to be the best configuration to control translational vibrations. However, to the best of the authors' knowledge, for the control of translational vibrations, the definition of such an omnidirectional TLCD is missing.

This study introduces the mathematical description and optimization scheme for omnidirectional tuned liquid column dampers (O-TLCDs) in the class of passive structural control, which can cover every translational vibration in all directions with full capacity and regardless of the excitation angle of incidence. In Section 2, the governing equation of motion is derived based on the Lagrange's principle, which is independent of the number of liquid columns and employs only a single degree of freedom. The independence of the O-TLCD from the excitation direction is analytically proven where the resulted damping of the O-TLCD is formulated by the non-conservative forces. In Section 3, to design an O-TLCD, an optimization scheme is introduced, which also accommodates a simulation of coupled O-TLCD-structure system under an arbitrary excitation. In Section 4, four O-TLCDs with different mass ratios are designed and investigated numerically under earthquake records, free vibration, harmonic excitation, and white noise. The performance of the O-TLCD, in general, and the role of critical parameters such as the mass ratio, the head loss coefficient, the liquid deflection and the excitations, in particular, are numerically investigated. In Section 5, experimental investigations are performed to study the validity of the proposed mathematical modeling approach, particularly focusing on the prediction of the natural frequency and the liquid deflection response. Finally, this paper is concluded in Section 6.

\section{Mathematical model}

This section introduces the equations of motion of the O-TLCD and the O-TLCD-structure system. In the following, the geometric layout and the DoF of the O-TLCD will be identified. 
Accordingly, the equations of motion will be derived based on Lagrange's principle and equilibrium of forces.

\subsection{Geometry of $O-T L C D$}

A TLCD with a horizontal liquid length $H$ and vertical liquid length $V$ is assumed, which is located in the direction of a translational excitation $w$ with arbitrary angle $\alpha$ relative to a coordinate system. It is also considered that the TLCD has the same cross sectional area $A$ in horizontal and vertical tubes. When this TLCD divides into two halves, which are called here as L-shape parts (L-arms), and $n$ number of L-arms are arranged symmetrically around an origin, an omnidirectional TLCD (O-TLCD) is created. Accordingly, the horizontal column of each L-arm is $H / 2$ and the angle between L-arms is $\psi=2 \pi / n$. With this definition where $n \in Z$, it is highlighted that TLCDs correspond to $n=2$ while O-TLCDs to $n \geq 3$. Fig. 1 represents the basic layout of an O-TLCD with three columns.

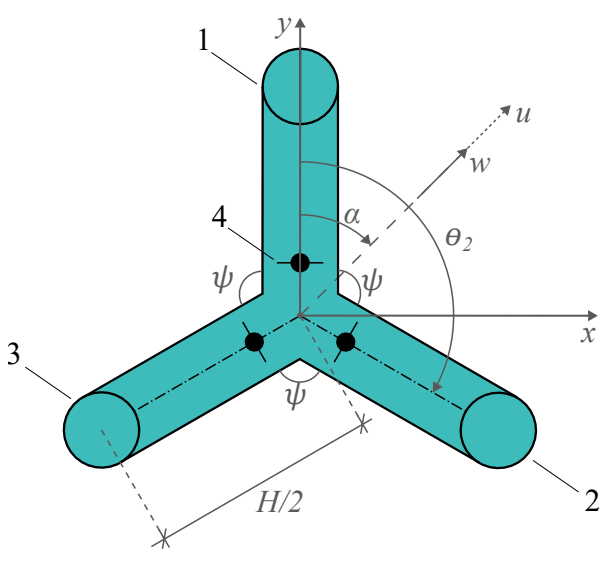

(a) Plan view

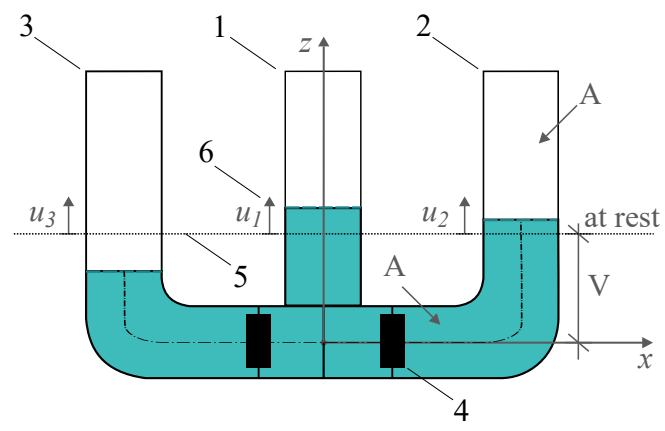

(b) Front view

Figure 1: An omnidirectional tuned liquid column damper (O-TLCD) with three columns, in the plan (a) and front view (b), where 1 to 3 are the three liquid columns, 4 is the orifice, 5 is the liquid level at rest and $u_{1}$ to $u_{3}(6)$ show the positive direction of liquid deflection in each column (the deflections are drawn considering the shown excitation $w)$.

\subsection{Degree of freedom of $O-T L C D$}

To develop both the motion equation of an O-TLCD and the interaction equation of an OTLCD-structure system, the liquid movement of each individual L-arm is required. For this purpose, the excitation angle $\alpha$ is considered with respect to the first tube. We consider a pseudo (imaginary) TLCD, which is representing the instantaneous damping effect of an O-TLCD with two L-arms and so a horizontal liquid length of $2 \times H / 2=H$ in the excitation direction. Then, we denote the liquid movement of the assumed TLCD by $u$ (Fig. 2a and 2b). Here, it is considered that the positive vertical liquid movement points to positive $z$ direction. It is also noted that the vertical and horizontal liquid movements (i.e. displacements, velocities and accelerations) are the same because of the assumption of the same cross sectional areas.

Projecting the liquid movement $u$, which is a pseudo deflection, onto each tube of the O-TLCD, one can find the liquid movement of $i$ th tube $u_{i}$ as

$$
u_{i}=u \cos \left(\alpha-\theta_{i}\right) \text {, }
$$



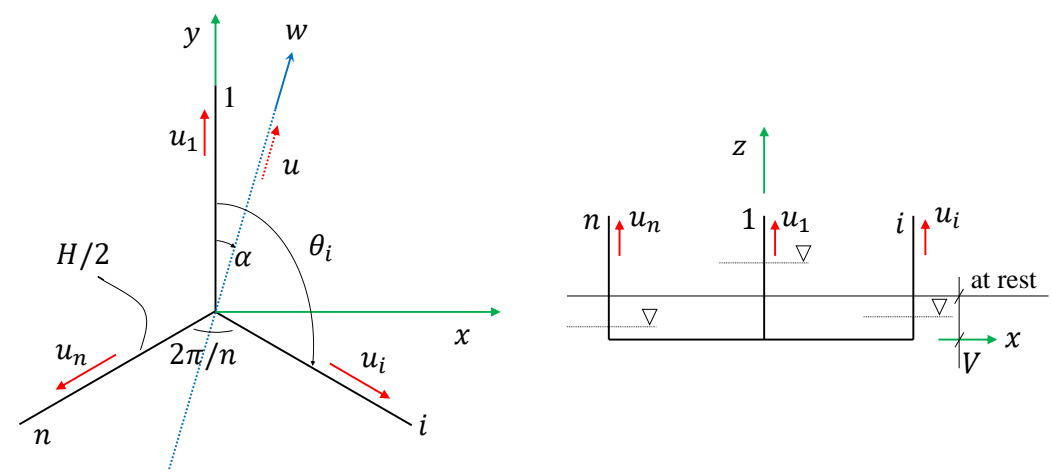

(a) plan and front view

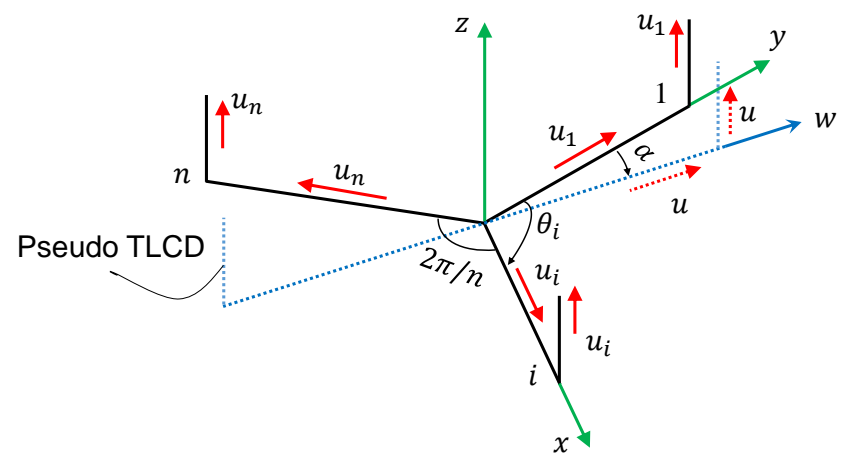

(b) $3 \mathrm{D}$ view

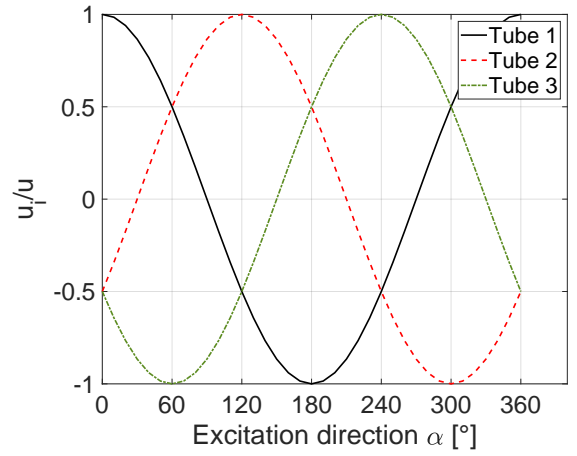

(c) Tubes' contribution

Figure 2: Graphical representation of the DoF of an O-TLCD and its TLCD representative with the equivalent damping effect in the excitation direction $\alpha$ with horizontal liquid length of $H(\mathrm{a}, \mathrm{b})$; the contribution of each tube to the liquid movement $u$ depending on $\alpha$ in a typical 3-Larm O-TLCD (c).

where $\theta_{i}$ represents the location of the $i$ th L-arm with respect to the first L-arm and is defined as

$$
\theta_{i}=2(i-1) \pi / n \text {. }
$$

when $\alpha$ is in terms of radian.

It is noted that, by using the pseudo TLCD, Eq. 1 represents O-TLCDs, regardless of number of L-arms, as a single degree of freedom system (SDoF). Eq. 1 is the key relation in mathematical description of O-TLCDs since it determines both the flow share and flow direction in each L-arm and that only based on $u$. It is evident that positive and negative values for $u_{i}$ in Eq. 1 demonstrate upward and downward liquid motions, respectively. As an example, Fig. 2c illustrate the liquid movements in the columns of an O-TLCD with three L-arms. It can be proven that a sum over all $n$ L-arms in Eq. 1, the total liquid movements, is zero which was already expected physically as the amount of liquid is constant:

$$
\sum_{i=1}^{n} u_{i}=u \sum_{i=1}^{n} \cos \left(\alpha-\theta_{i}\right)=0 .
$$




\subsection{Equation of motion of $O-T L C D$}

To develop the equation of motion of the $n$-L-arm O-TLCD, the Lagrange's principle is employed:

$$
\frac{\mathrm{d}}{\mathrm{dt}}\left(\frac{\partial E_{k i n}}{\partial \dot{u}}\right)-\frac{\partial E_{k i n}}{\partial u}+\frac{\partial E_{p o t}}{\partial u}=Q_{u}
$$

where $E_{k i n}$ is the kinetic energy, $E_{p o t}$ is the potential energy and $Q_{u}$ is the non-conservative force. Unless otherwise mentioned, it is assumed that $n \geq 2$ in the following. Furthermore, it is considered the geometric parameters, such as $A$ are constant and do not vary with time.

\subsubsection{Kinetic energy}

The kinetic energy of an O-TLCD with $n$ L-arms can be written as follows:

$$
E_{k i n}=\frac{1}{2} \rho A \sum_{i=1}^{n}\left(\frac{H}{2}\left|\dot{\mathbf{u}}_{H, i}\right|^{2}+V\left|\dot{\mathbf{u}}_{V, i}\right|^{2}\right)
$$

where $\rho$ is the liquid density; $\dot{\mathbf{u}}_{H, i}$ and $\dot{\mathbf{u}}_{V, i}$ are the total liquid velocity vectors in the horizontal and the vertical parts of the $i$ th L-arm, respectively, which, for the case $\alpha$ is a constant, can be defined considering the magnitude of the relative liquid velocity $\dot{u}_{i}=\dot{u} \cos \left(\alpha-\theta_{i}\right)$ and the magnitude of excitation velocity $\dot{w}$ (see Eq. 1 and Fig. 2 ) as

$$
\begin{gathered}
\dot{\mathbf{u}}_{V, i}^{\mathrm{T}}=\left[\begin{array}{lll}
\dot{w} \sin \alpha & \dot{w} \cos \alpha & \dot{u} \cos \left(\alpha-\theta_{i}\right)
\end{array}\right], \\
\dot{\mathbf{u}}_{H, i}^{\mathrm{T}}=\left[\begin{array}{lll}
\dot{u} \cos \left(\alpha-\theta_{i}\right) \sin \theta_{i}+\dot{w} \sin \alpha & \dot{u} \cos \left(\alpha-\theta_{i}\right) \cos \theta_{i}+\dot{w} \cos \alpha & 0
\end{array}\right],
\end{gathered}
$$

which bring,

$$
E_{k i n}=\frac{1}{2} \rho A\left(\left(\frac{H}{2}\left(\dot{u}^{2}+2 \dot{u} \dot{w}\right)+V \dot{u}^{2}\right) \sum_{i=1}^{n} \cos ^{2}\left(\alpha-\theta_{i}\right)+n \dot{w}^{2}\left(\frac{H}{2}+V\right)\right)
$$

and

$$
\frac{\mathrm{d}}{\mathrm{dt}}\left(\frac{\partial E_{k i n}}{\partial \dot{u}}\right)=\frac{1}{2} \rho A(H(\ddot{u}+\ddot{w})+2 V \ddot{u}) \sum_{i=1}^{n} \cos ^{2}\left(\alpha-\theta_{i}\right) .
$$

It is noted that for the case of the time-variant $\alpha, \dot{u}_{i}=\dot{u} \cos \left(\alpha-\theta_{i}\right)-\dot{\alpha} u \sin \left(\alpha-\theta_{i}\right)$ should be considered for calculating the kinetic energy in Eq. 5. However, for the sake of simplicity in the following, we consider that $\alpha$ is constant during an excitation event.

\subsubsection{Potential energy}

The potential energy comes from the influence of the gravity on the liquid movement in vertical tubes:

$$
E_{\text {pot }}=\sum_{i=1}^{n} \frac{1}{2} \rho g A\left(V+u \cos \left(\alpha-\theta_{i}\right)\right)^{2} .
$$


Expanding the squared term and using Eq. 3, one can end up with

$$
\begin{gathered}
E_{\text {pot }}=\frac{1}{2} \rho g A\left(n V^{2}+u^{2} \sum_{i=1}^{n} \cos ^{2}\left(\alpha-\theta_{i}\right)\right), \\
\frac{\partial E_{p o t}}{\partial u}=\rho g A u \sum_{i=1}^{n} \cos ^{2}\left(\alpha-\theta_{i}\right) .
\end{gathered}
$$

\subsubsection{Non-conservative force, $Q_{u}$}

In fluid dynamics, the non-conservative force of a pipe portion can be computed using the pressure loss $\Delta p_{L}$ and pipe cross section $A$ at the flow region under consideration:

$$
Q_{u}=-\Delta p_{L} A,
$$

The $\Delta p_{L}$ depends on the stagnation pressure $\rho v^{2} / 2$ and loss factor $\lambda(R e)$ :

$$
\Delta p_{L}=\rho \frac{v^{2}}{2} \lambda(R e)
$$

where the term $\rho v^{2} / 2$ is also known as dynamic pressure [55]. The pressure loss $\Delta p_{L}$ reduces to the following form for turbulent flow ( $R e \gtrsim 4000$ in circular pipes)

$$
\Delta p_{L}=\rho \frac{v^{2}}{2} \lambda,
$$

where $v$ is the liquid flow velocity and $\lambda$ is the loss factor which is here independent of Reynolds number Re.

The dynamic pressure $\rho v^{2} / 2$ represents the pressure rise when the fluid in motion is brought to a stop isentropically, which occurs at the so called stagnation point [55]. The O-TLCD presents $n$ flow regions where the liquid in each horizontal tube can reach a stagnation point. Accordingly, the total non-conservative force of O-TLCD can be represented as the sum of non-conservative forces in all L-arms. Therefore, the sum of non-conservative forces in all horizontal tubes can be considered as

$$
Q_{u}=-\sum_{i=1}^{n} Q_{u, i}=-\sum_{i=1}^{n} A \rho \lambda \frac{\dot{u}_{i}^{2}}{2}=-\frac{1}{2} A \rho \lambda \dot{u}^{2} \sum_{i=1}^{n} \cos ^{2}\left(\alpha-\theta_{i}\right) \quad n \geq 3 \quad \text { (O-TLCD). }
$$

For TLCD, on the other hand, the non-conservative force is defined in literature as (e.g. see Hochrainer [56])

$$
Q_{u}=-\frac{1}{2} A \rho \lambda \dot{u}^{2} \cos ^{2}\left(\alpha-\theta_{i}\right) \quad n=2 \quad(\mathrm{TLCD}),
$$

which, here, is extended to the case where the excitation is not aligned with the TLCD.

Introducing Eq. 9, Eq. 12, Eq. 16, and Eq. 17 into Eq. 4, one can end up with the equation of motion of TLCDs $(n=2)$ and $n$-L-arm O-TLCDs $(n \geq 3)$ with identical pipe cross sections as follows:

$$
\ddot{u}+\delta|\dot{u}| \dot{u}+\omega_{d}^{2} u=-\gamma \ddot{w},
$$


where the effective length $L$, the natural frequency $\omega_{d}$, and the geometric factor $\gamma$ are defined as

$$
L=2 V+H \quad \omega_{d}=\sqrt{\frac{2 g}{L}} \quad \gamma=\frac{H}{L}
$$

and the head loss coefficient $\delta$ as

$$
\delta=\left\{\begin{array}{lll}
\frac{1}{2 L} \lambda & \text { if } n=2 & (\mathrm{TLCD}) \\
\frac{1}{L} \lambda & \text { if } n \geq 3 & (\mathrm{O}-\mathrm{TLCD})
\end{array}\right.
$$

The solution of Eq. 18 is the liquid deflection of the pseudo TLCD which is introduced in Fig. 2, Subsequently, the liquid deflection in the $i$ th L-arm of the O-TLCD is simply obtained using Eq. 1.

\subsection{Equation of motion of O-TLCD-structure systems}

This section presents the equation of motion of a structural system equipped with an O-TLCD (Fig. 3). A symmetric 3-dimentional SDoF structure, with the mass $m_{s}$, the stiffness coefficient $k_{s}$ and the damping coefficient $c_{s}$, is considered. The structure is excited by an external excitation $F$, which is applied at the mass center and in arbitrary constant direction $\alpha$. Accordingly, the floor moves with single degree of freedom $w$ in the direction $\alpha$.

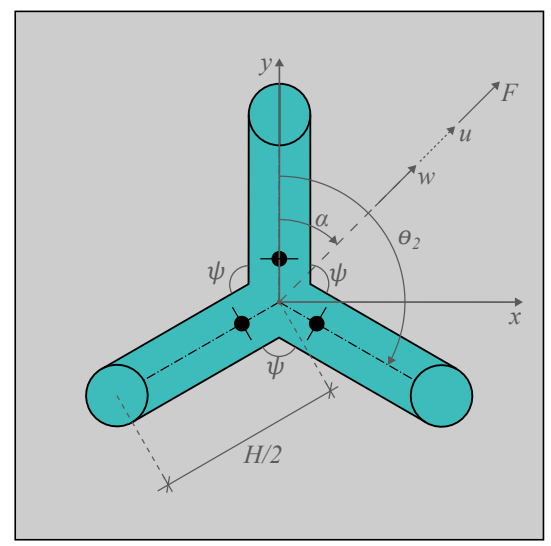

(a) Plan view

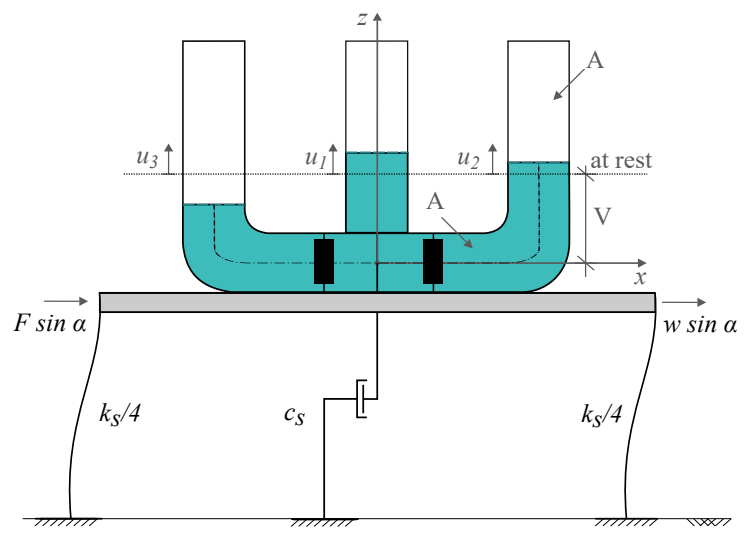

(b) Front view

Figure 3: Coupled O-TLCD-structure system in the plan (a) and front view (b), excited by an external excitation $F)$.

To derive the interaction equations, first, the active liquid mass $m_{a c t, i}$ of the $i$ th $\mathrm{L}$-arm is identified:

$$
m_{a c t, i}=\frac{H / 2}{n(V+H / 2)} m_{d}=\frac{1}{n} \gamma m_{d},
$$

which is the horizontal liquid portion of the total liquid mass $m_{d}$.

To calculate the resultant inertial forces of the O-TLCD, Eq. 1 is used to form the liquid acceleration vector of all $n$ L-arms:

$$
\ddot{\mathbf{u}}^{T}=\left[\ddot{u} \cos \left(\alpha-\theta_{1}\right) \quad \ddot{u} \cos \left(\alpha-\theta_{2}\right) \ldots \ddot{u} \cos \left(\alpha-\theta_{n}\right)\right] .
$$




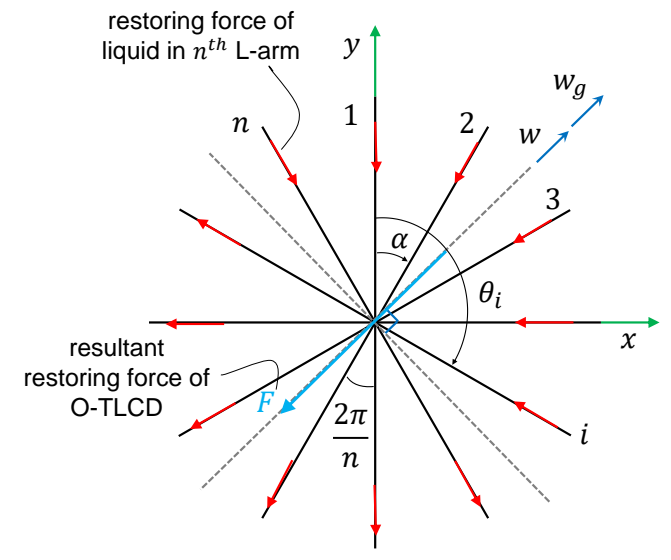

(a) Restoring force distribution

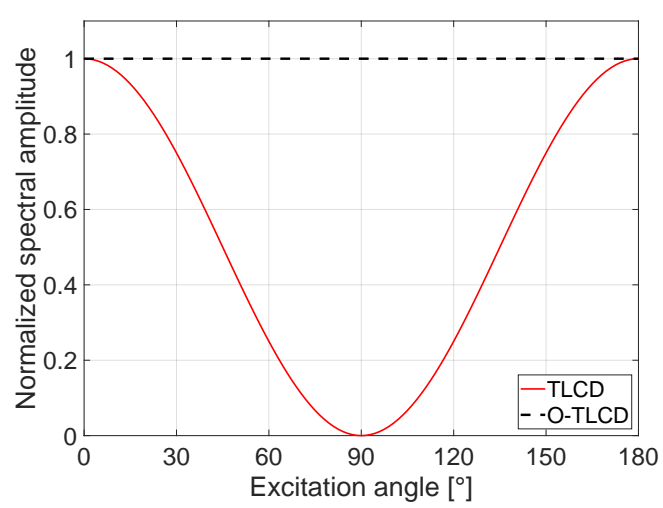

(b) O-TLCD vs. TLCD

Figure 4: Distribution of the restoring force exerted by a typical O-TLCD to a structure (a); comparison between the performance of the O-TLCD and the TLCD when the excitation angle varies (b).

Furthermore, since the resultant inertial force of the O-TLCD is in the excitation direction, a vector $\mathbf{p}$ is defined to project the liquid motion of each tube onto this direction:

$$
\mathbf{p}^{T}=\left[\begin{array}{lll}
\cos \left(\alpha-\theta_{1}\right) & \cos \left(\alpha-\theta_{2}\right) \ldots \cos \left(\alpha-\theta_{n}\right)
\end{array}\right] .
$$

Then, the equation of motion of the O-TLCD-structure system can be obtained based on equilibrium of forces in the excitation direction and using the elements of vectors $\ddot{\mathbf{u}}$ and $\mathbf{p}$ (Fig. 4a):

$$
\left(m_{s}+m_{d}\right) \ddot{w}+c_{s} \dot{w}+k_{s} w+\sum_{i=1}^{n}\left(m_{a c t, i} \ddot{u} \cos ^{2}\left(\alpha-\theta_{i}\right)\right)=F .
$$

To finalize Eq. 24, it is necessary to differentiate TLCDs with $n=2$ from O-TLCDs with $n \geq 3$ in calculating the summation. For TLCDs, we have

$$
\sum_{i=1}^{n} \cos ^{2}\left(\alpha-\theta_{i}\right)=2 \cos ^{2} \alpha \quad n=2 \quad(\mathrm{TLCD}) .
$$

For O-TLCDs, on the other hand, it can be proven that

$$
\sum_{i=1}^{n} \cos ^{2}\left(\alpha-\theta_{i}\right)=\frac{n}{2} \quad n \geq 3 \quad(\mathrm{O}-\mathrm{TLCD}) .
$$

Applying the Eq. 25, Eq. 26 and Eq. 21 to Eq. 24, the interaction equation of motion can be written as

$$
m_{s} \ddot{w}+c_{s} \dot{w}+k_{s} w=F-m_{d} \ddot{w}-q \gamma m_{d} \ddot{u},
$$

or equivalently

$$
\ddot{w}+2 D_{s} \omega_{s} \dot{w}+\omega_{s}^{2} w=f-\mu(\ddot{w}+q \gamma \ddot{u}),
$$


for

$$
q= \begin{cases}\cos ^{2} \alpha & \text { if } n=2 \\ \frac{1}{2} & \text { if } n \geq 3\end{cases}
$$

where $f=F / m_{s}$ mass-normalized external force, $D_{s}$ is the damping coefficient and $\omega_{s}$ is the natural frequency of the main structure; $\mu$ is the mass ratio between the liquid mass and the mass of the structure. It is noted that, in Eq. 28, the movement of the structure in the $x$ and the $y$ direction can be simply obtained by $w \sin \alpha$ and $w \cos \alpha$, respectively; the liquid acceleration $\ddot{u}$ in the path of the excitation comes from Eq. 18. Furthermore, if the external excitation is a ground motion applied at the projected mass center of the structure on the ground in $\alpha$ direction, the equations of motion in Eq. 18 and in Eq. 28 can be presented as

$$
\begin{gathered}
\ddot{u}+\delta|\dot{u}| \dot{u}+\omega_{d}^{2} u=-\gamma\left(\ddot{w}+\ddot{w}_{g}\right), \\
\ddot{w}+2 D_{s} \omega_{s} \dot{w}+\omega_{s}^{2} w=-\ddot{w}_{g}-\mu\left(\ddot{w}+\ddot{w}_{g}+q \gamma \ddot{u}\right),
\end{gathered}
$$

where $\ddot{w}_{g}$ is the ground acceleration.

Eq. 28 (or Eq. 31) states that, in contrast to TLCDs whose counteracting force is $\alpha$-dependent as expected, by employing a symmetric O-TLCD with 3 or greater number of L-arms, the interaction equation of motion is independent of the excitation angle $\alpha$ when $\alpha$ is not changing during the excitation event. This is significant since O-TLCDs can exert identical counteracting forces in all directions to the structure regardless of the excitation angle. For the sake of simplicity, as mentioned before, we present here the equations for the excitation direction, which is not changing during the excitation event. If $\alpha$ is changing, the Lagrange formulation with its components can be expanded as well.

Concerning the mass ratio in Eq. 28 (or Eq. 31) which is generally defined as

$$
\mu=\frac{n \rho A(V+H / 2)}{m_{s}},
$$

it is important to note that increasing the number of L-arms $n$ increases the active mass ratio $\mu^{*}$, which is defined in analogy with TMDs by introducing $u^{*}=u / \gamma$ [56] into the coupled equations of motion in Eq. 18 and Eq. 28 (or Eq. 30 and Eq. 31):

$$
\mu^{*}=\frac{\mu \gamma^{2}}{1+\mu\left(1-\gamma^{2}\right)},
$$

which in turn decreases the optimal frequency $f_{d, o p t}$ (proposed by Warburton [57] for TMDs without superscript $\left.{ }^{*}\right)$, which can be used for the O-TLCD as

$$
f_{d, o p t}=f_{s}^{*} \frac{\sqrt{1-\mu^{*} / 2}}{1+\mu^{*}},
$$

where based on the increased mass of the structure $m_{s}^{*}$ due to the dead mass of the liquid:

$$
m_{s}^{*}=m_{s}\left(1+\mu\left(1-\gamma^{2}\right)\right),
$$

$f_{s}^{*}$ can be obtained out of the natural frequency of the main structure $f_{s}$ :

$$
f_{s}^{*}=f_{s} \sqrt{\frac{m_{s}}{m_{s}^{*}}} .
$$


Eq. 34 shows that, according to Eq. 19, while changing the number of L-arms $n$ does not affect the natural frequency of the O-TLCD, the change in $n$ may demand retuning the O-TLCD, which results in a different geometry. Therefore, it is generally not simple to directly find the influence of number of L-arms on the control force in Eq. 28 and, for example, find a clear comparison between the magnitude of control forces produced by O-TLCDs and TLCDs. However, to provide a relative comparison, it is considered here that O-TLCDs and TLCDs are designed individually and optimally to work with full capacity for a single system. Accordingly, the performance of each system can be normalized to their maximum control force and recorded for different incremental excitation angle from 0 to $180^{\circ}$, which is illustrated in Fig. $4 \mathrm{~b}$,

\section{Design criteria and optimization scheme}

This paper proposes a constrained optimization scheme (Fig. 5) to design the O-TLCD. The tuning criteria proposed by Warburton [57, which is basically the extension of the criteria of Den Hartog [58] for harmonic vibrations to random excitations, is employed in this approach. Using Warburton's formulas for TMDs and the definition of active mass ratio in Eq. 33, the optimal frequency and the optimal damping ratio for O-TLCDs are calculated by Eq. 34 and Eq. 37 for undamped structures, respectively.

$$
D_{d, o p t}=\sqrt{\frac{\mu^{*}\left(1-\mu^{*} / 4\right)}{4\left(1+\mu^{*}\right)\left(1-\mu^{*} / 2\right)}} .
$$

The tuning constrains of the optimization scheme are defined as

$$
\frac{f_{d}-f_{d, o p t}}{f_{d, o p t}} \leq \Delta_{f}, \quad D_{d, o p t} \geq D_{d, \min },
$$

where $f_{d}=\omega_{d} / 2 \pi$ is the natural frequency of the O-TLCD; $\Delta_{f}$ is the tolerance value, for which 0.01 , and $D_{d, \min }$ is the minimum desired optimal damping, for which 0.05 is recommended. For comparison with optimal damping ratio in Eq. 37, the damping ratio of the O-TLCD is approximated by a linearized viscose damping ratio used for harmonic vibrations [25]:

$$
D_{d, l i n}=\frac{4 U_{0} \delta}{3 \pi}
$$

where $U_{0}$ is the liquid vibration amplitude.

The mass ratio $\mu$, defined by Eq. 32 , is constrained by a plausible upper limit $\left(\mu_{\max }\right)$ with respect to the total mass of the primary structure that is either known or can be conservatively estimated based on the modal mass. Concerning the geometry, an O-TLCD with rectangular cross section, which is easier to be manufactured than the circular one and brings the advantage of using different dimensions for providing the cross section $A$, is considered. To find an optimum and feasible geometry a set of linear and nonlinear geometric constraints are defined, for which Fig. 6 introduces the geometric parameters that facilitates the description of the constraints.

In this figure, parameter $s$, which is equal to $\left(H_{i}-\frac{t_{V}}{2}\right)$, indicates the net horizontal column of each L-arm and together with the half of the vertical column width $t_{V}$ determines the outer horizontal length of the L-arm in O-TLCDs, while the $H_{i}$ determines the centered one. It can be found from Fig. 6 that the parameter $s$ depends on the width of each L-arm $b$, the angle between 


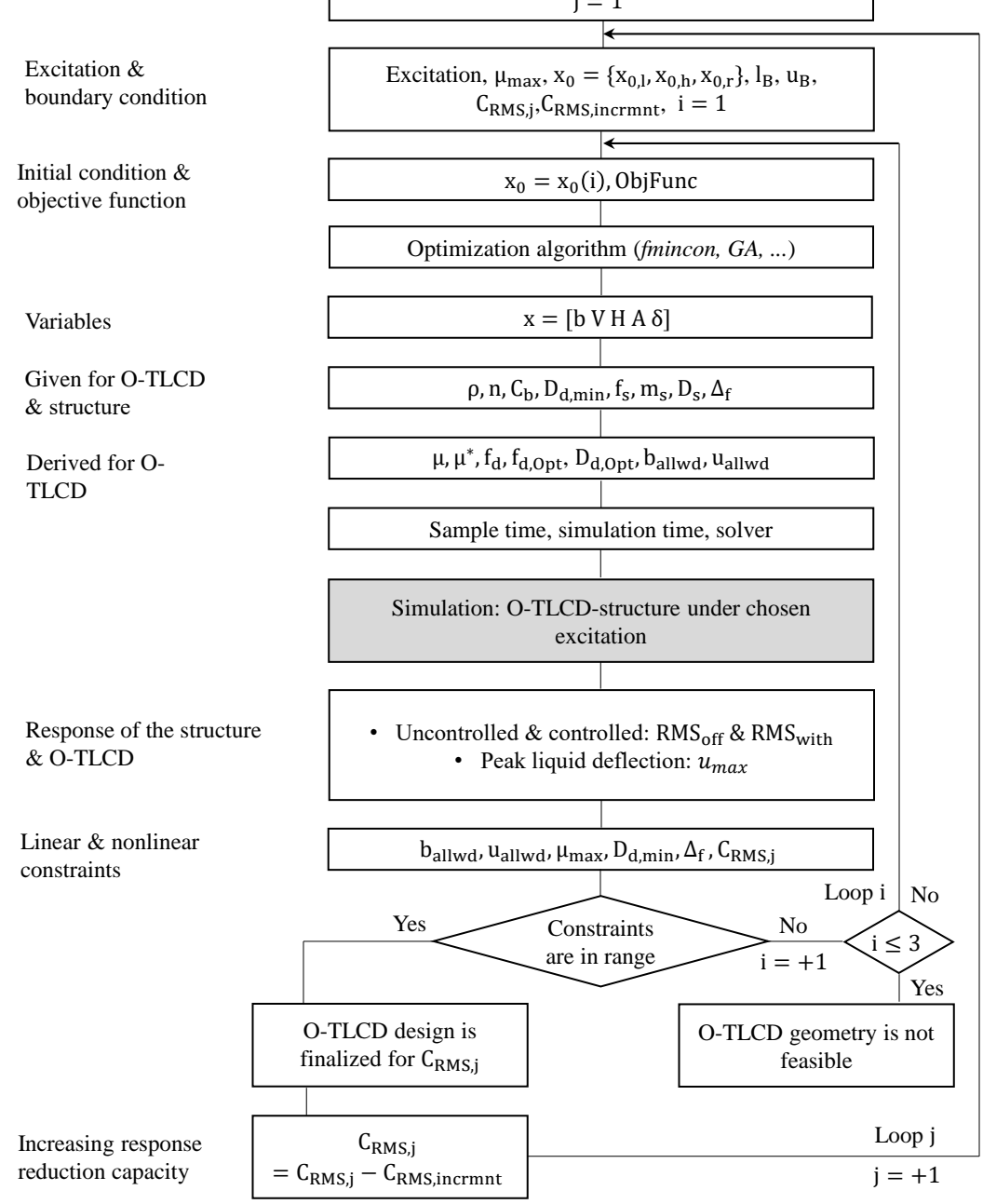

Figure 5: The proposed optimization scheme for designing O-TLCDs under an arbitrary excitation.

L-arms $\psi$ (i.e. also the number of L-arms $n$ ), $t_{V}$ and $H_{i}$. Accordingly, in order to achieve a desirable space for the horizontal column length of each L-arm, the following constraint can be defined

$$
b \leq C_{b} \frac{2(1-\cos \psi)}{\sin \psi}\left(H_{i}-\frac{t_{V}}{2}\right)
$$

where $C_{b}$ is a user-defined coefficient in $[0,1]$ interval; in practice, $C_{b} \leq 0.50$ results in a reasonable geometry. The right hand side of Eq. 40 is introduced as $b_{\text {allwd }}$ which defines an upper design limit for $b$ and will be used for the optimization scheme.

The other important constrain comes from the responses of the primary structure and the OTLCD. In the optimization scheme in each loop $(j, i)$, simulation of the resulted coupled system using desirable sample and simulation time and a solver type as numerical integration method is performed online. The RMS of the response of the controlled structure (the structure with OTLCD) $R M S_{\text {with }}$ is constrained to be smaller than the RMS of the response of the uncontrolled structure (the structure with off O-TLCD whose liquid movement is suppressed despite existence) 


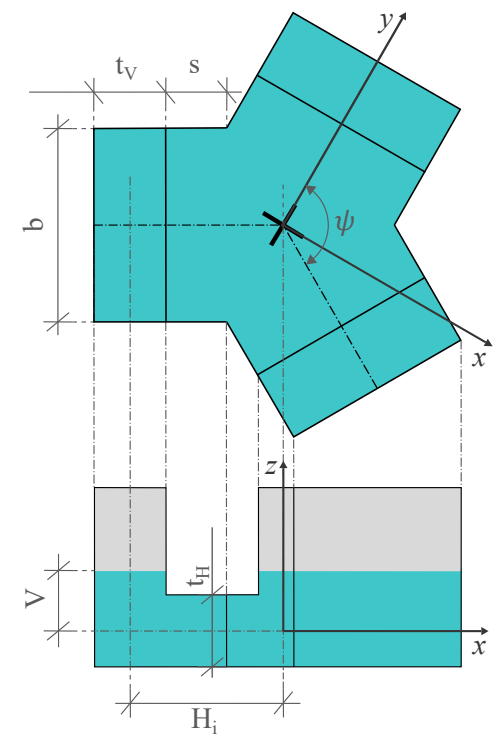

Figure 6: Geometric parameters of the O-TLCD used in the optimization scheme.

$R M S_{\text {off }}$ under arbitrarily chosen excitation. More precisely,

$$
\frac{R M S_{w i t h}}{R M S_{\text {off }}} \leq C_{R M S}
$$

where $C_{R M S}$ is a user-defined upper limit in the $[0,1]$ interval. Although the value for $C_{R M S}$ depends on the intensity of the excitation and the desire of the control designer, $C_{R M S}=0.50$ is recommended for reasonable O-TLCD geometries. Incrementally decreasing the $C_{R M S}$ by a predefined value $C_{R M S, \text { incrmnt }}$ in $j$ loops:

$$
C_{R M S, j}=C_{R M S, j}-C_{R M S, \text { incrmnt }},
$$

the algorithm finds the optimum O-TLCD geometry as long as the prescribed RMS upper limit at the $j$ th loop, $C_{R M S, j}$, can be achieved.

Furthermore, the considered equation of motion of O-TLCD would no longer be valid if the liquid deflection $u$ is larger than the theoretical liquid height $V$ in the vertical column [17. Therefore, in the optimization scheme, the maximum liquid deflection $u_{\max }$ under arbitrarily chosen excitation is constrained - to be in the safe side - not only to $V$ but rather to the net liquid height located immediately above the horizontal column $u_{\text {allwd }}=V-t_{H} / 2$.

To implement the proposed optimization scheme, the minimum of constrained nonlinear multivariable (fmincon) function in Matlab is employed; it is worth mentioning that other optimization algorithms such as genetic algorithm (GA) is applicable in the proposed scheme as well. Five parameters $b, V, H_{i}, A$ (see Fig. 1) and $\delta$ (see Eq. 20) are selected as the independent unknown variables and the $\gamma$, defined in Eq. 19, is considered as the objective function to be maximized.

Finally, it should be highlighted that, in order to fairly compensate the role of the initial point $\left(x_{0}\right)$ in the minimization process which may lead to local minima, the developed scheme can initialize the problem at least from three different origins. Two of these points are clearly higher $\left(x_{0, h}\right)$ and lower $\left(x_{0, l}\right)$ estimated initial guesses and the third one is a random initial point $\left(x_{0, r}\right)$ 
generated based on either the first or the second chosen initial point. The initial points are checked successively in $i$ loops.

The outcome of the described scheme is a unique geometry for the O-TLCD optimized when the coupled O-TLCD-structure system is exposed to an arbitrary excitation, such as an earthquake, with a particular intensity. In this system, the same O-TLCD can be still utilized under other excitations with higher intensities and different content as long as the liquid deflection does not violate $u_{\text {allwd }}$, which can be possibly achieved by increasing the head loss coefficient $\delta$ in Eq. 20 . On the other hand, it can be also the case under weaker excitations for which lower $\delta$ might be required to employ the advantage of higher liquid deflection for more vibration mitigation during the excitation. However, it should be ascertained that the manipulation of the head loss coefficient requires a semi-active control of the O-TLCD, which is beyond the scope of the current study.

\section{Numerical Investigations}

A seismically excited SDoF structure with an O-TLCD is investigated. To evaluate the performance of O-TLCDs, a symmetric high-rise structure (Fig. 7) with natural frequency $f_{s}=0.280 \mathrm{~Hz}$ is taken into account. Furthermore, it is assumed that the first modal mass is $m_{s}=400 \mathrm{t}$ and the first modal damping ratio is $D_{s}=1 \%$

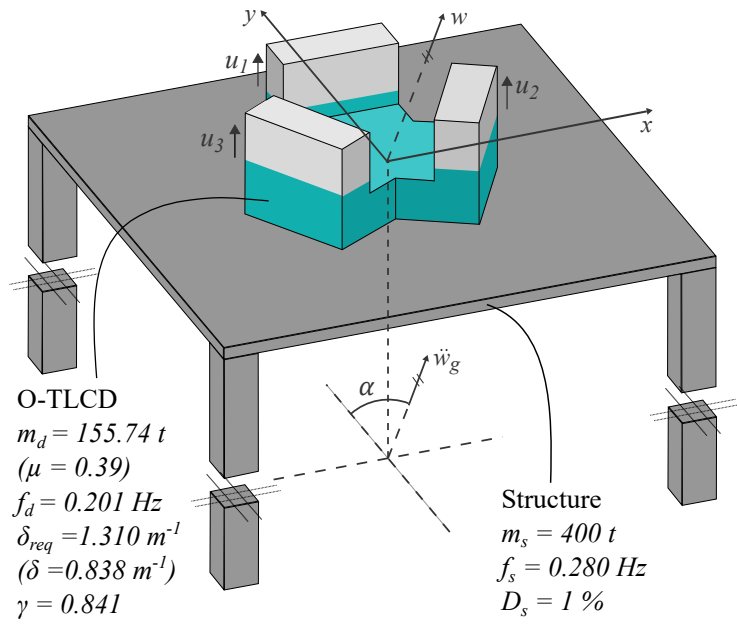

(a)

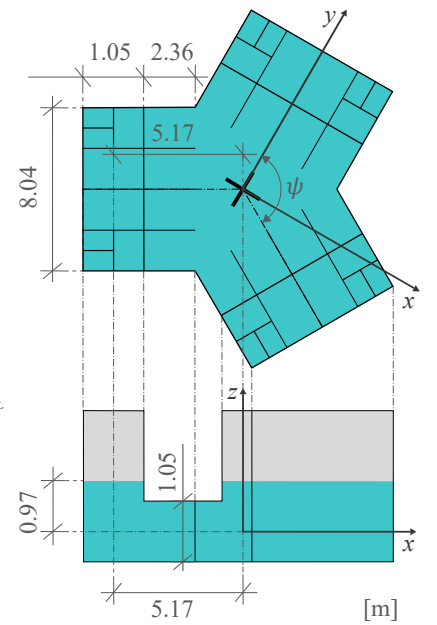

(b)

Figure 7: The example structure controlled by the O-TLCD (a) and the plan and the side view of one of the designed O-TLCDs $(\mu=0.39)(\mathrm{b})$.

In the following, first, the optimized O-TLCDs using different mass ratios are designed, which function with optimum head loss coefficient $\delta$ for the described structure under a particular earthquake. Next, the coupled O-TLCD-structure systems are evaluated under an ensemble of earthquakes, for which the general need for a higher head loss coefficient $\delta_{r e q}$ is presented. The O-TLCDs equipped with $\delta_{r e q}$ are further investigated under a free vibration, harmonic excitation and white noise, subsequently.

\subsection{Optimized $O-T L C D$}

To design the O-TLCD, four mass ratios ranging from $\mu=10$ to $40 \%$ with respect to the first modal mass of the structure are chosen as the mass-ratio upper-limits in the optimiza- 
tion scheme. Estimating a total mass of such structure to be, for example, three times the first modal mass, the investigated mass ratios cover a quite reasonable range with respect to the total mass of the structure. Other constrains include $\Delta_{f}=0.01, \Delta_{D}=0.1, C_{b}=0.50$, $C_{R M S, 1}=0.50, C_{R M S, \text { incrmnt }}=0.01$, and lower bound $l_{B}=[1,0.1,1,0.1,0.01]$ and upper bound $u_{B}=[100,10,100,10,10]$ for the five unknown geometric variables $\left[b, V, H_{i}, A, \delta\right]$ with the corresponding units as $[\mathrm{m}]$ for $b, V, H_{i},\left[\mathrm{~m}^{2}\right]$ for $A$, and $\left[\mathrm{m}^{-1}\right]$ for $\delta$. To compute the RMS value in Eq. 41. the displacement response of the structure is utilized.

The earthquake record Kobe (1995) with the intensity factor of one is utilized for the design of each of four O-TLCDs using the optimization scheme described in the last section. Therefore, as the Kobe record is used in the optimization, the El Centro (1940), Hachinohe (1968) and Northridge (1994) earthquakes are opted for the validation phase. Fig. 8 shows the earthquake records. The simulations are conducted using the ode8 (Dormand-Prince) time integration method in Simulink/Matlab. The simulation time of each calculation is $150 \mathrm{~s}$ with a time increment of $0.01 \mathrm{~s}$ for Hachinohe and $0.02 \mathrm{~s}$ for other records.

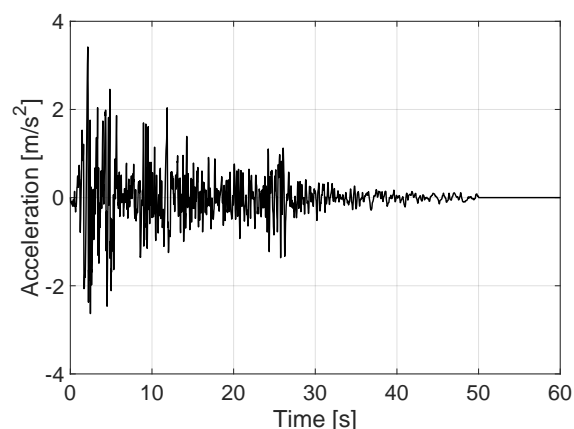

(a) El Centro (1940)

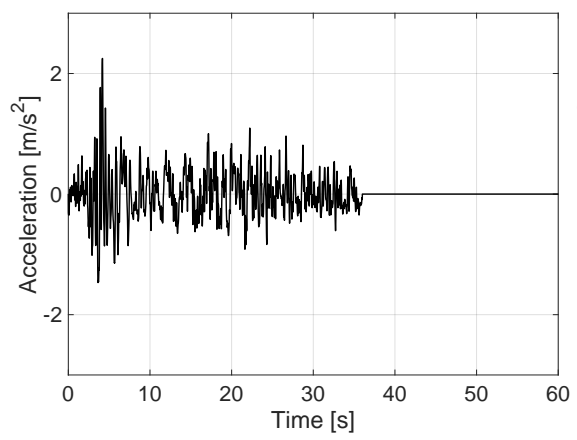

(c) Hachinohe (1968)

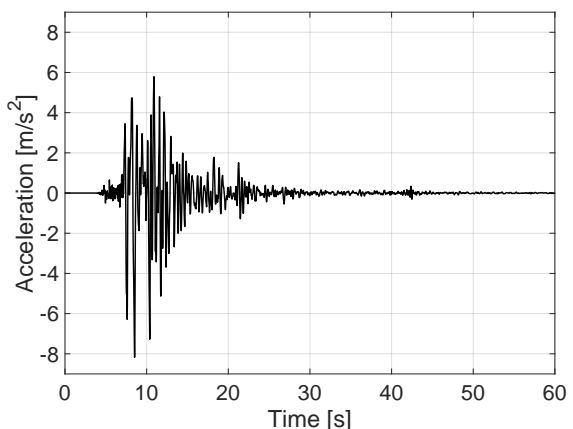

(b) Kobe (1995)

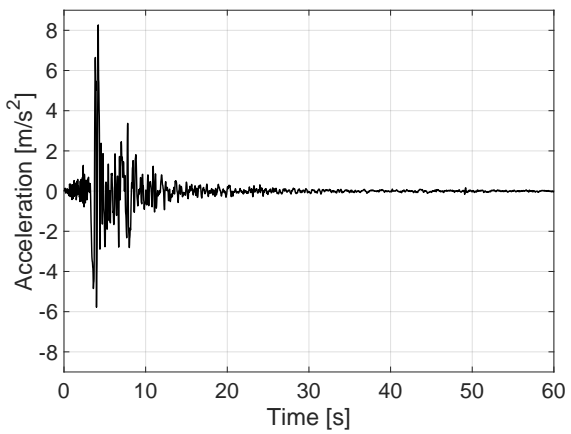

(d) Northridge (1994)

Figure 8: Time-history plots of earthquake records used in this study.

Table 1 and 2 summarize the outcomes of the optimization scheme for the aforementioned four mass-ratio upper-limits in two parts and Fig. 9 depicts the obtained geometries. First of all, searching for the most optimized geometry, it is noted in the table and the figure that the optimization scheme exploited the mass-ratio upper-limits fully for all cases except for the $40 \%$ limit. This implies that no larger tuned geometry for O-TLCD, which can attenuate uncontrolled responses more than $C_{R M S}=0.25$, exists to accomodate liquid mass more than $0.389 \mathrm{~m}_{s}$. Therefore, it is confirmed that the developed optimization scheme is able to converge to the most plausible and 
efficient O-TLCD for the structure under consideration, which, according to the indices $\mu^{*}, D_{d, \text { opt }}$, $D_{d, l i n}, \gamma$, and $C_{R M S}$ in Table 1 and 2 , is the O-TLCD ${ }_{\mu=0.39}$.
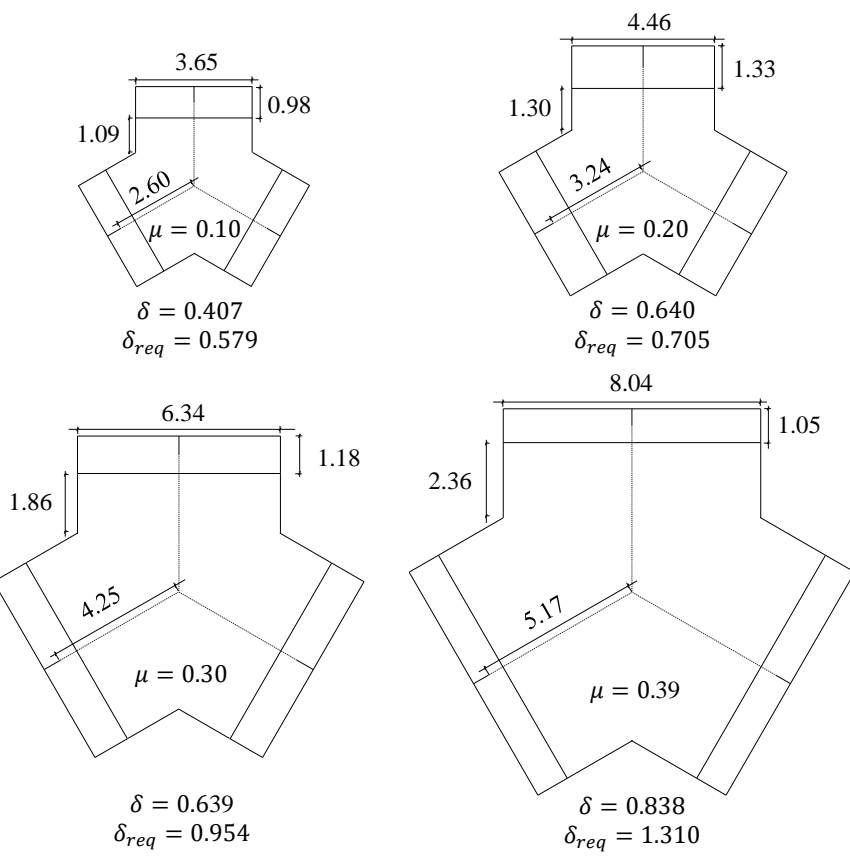

Figure 9: Characteristics of the investigated O-TLCDs with different mass ratios (dimensions are in [m]; the optimal head loss coefficient $\delta$ and the required one $\delta_{r e q}$ are in $\left.\left[\mathrm{m}^{-1}\right]\right)$.

Table 1: Parameters (Part 1) of the designed O-TLCDs with different mass ratios $\mu$.

\begin{tabular}{ccccccccc}
\hline & $b[\mathrm{~m}]$ & $V[\mathrm{~m}]$ & $H[\mathrm{~m}]$ & $A\left[\mathrm{~m}^{2}\right]$ & $\delta\left[\mathrm{m}^{-1}\right]$ & $\delta_{r e q}\left[\mathrm{~m}^{-1}\right]$ & $\mu^{*}[-]$ & $m_{s}^{*}[-]$ \\
\hline$\mu=0.10$ & 3.65 & 1.21 & 5.20 & 3.58 & 0.407 & 0.579 & 0.05 & 421.92 \\
$\mu=0.20$ & 4.46 & 1.25 & 6.48 & 5.93 & 0.640 & 0.705 & 0.09 & 438.38 \\
$\mu=0.30$ & 6.34 & 1.11 & 8.50 & 7.46 & 0.640 & 0.950 & 0.17 & 444.63 \\
$\mu=0.39$ & 8.04 & 0.98 & 10.34 & 8.45 & 0.838 & 1.310 & 0.25 & 445.52 \\
\hline
\end{tabular}

Table 2: Parameters (Part 2) of the designed O-TLCDs with different mass ratios $\mu$.

\begin{tabular}{ccccccccc}
\hline & $f_{s}^{*}[\mathrm{~Hz}]$ & $f_{d, o p t}[\mathrm{~Hz}]$ & $f_{d}[\mathrm{~Hz}]$ & $D_{d, o p t}[-]$ & $D_{d, l i n}[-]$ & $u_{\text {allwd }}[\mathrm{m}]$ & $\gamma[-]$ & $C_{R M S}[-]$ \\
\hline$\mu=0.10$ & 0.273 & 0.258 & 0.255 & 0.10 & 0.18 & 0.72 & 0.68 & 0.46 \\
$\mu=0.20$ & 0.267 & 0.238 & 0.235 & 0.15 & 0.18 & 0.59 & 0.72 & 0.34 \\
$\mu=0.30$ & 0.266 & 0.217 & 0.215 & 0.19 & 0.21 & 0.52 & 0.79 & 0.27 \\
$\mu=0.39$ & 0.265 & 0.199 & 0.201 & 0.23 & 0.25 & 0.45 & 0.84 & 0.25 \\
\hline
\end{tabular}

Beside the geometries, Table 1 and Fig. 9 show the head loss coefficient $\delta$ coming from the optimization scheme under the selected earthquake Kobe for all mass ratios. However, since the occurrence of intenser excitations than Kobe is probable, these optimized head loss coefficients $(\delta)$ might not be sufficient to prevent the liquid motion from exceeding the provided liquid deflection 
$u_{\text {allwd }}$ which is also reported in Table 2 for each O-TLCD. This can be investigated when we simulate the performance of the presented O-TLCDs in controlling the structure in Fig. 7 under different excitations.

\subsection{Earthquake excitation, with $\delta$}

Fig. 10 and Fig. 11 show the response of the structure equipped with the O-TLCD ${ }_{\mu=0.10}$ and the O-TLCD ${ }_{\mu=0.39}$, which are the lightest and the heaviest O-TLCDs and present the lowest and the highest active mass ratios $\mu^{*}$ (Table 1), under all four earthquakes, respectively. In spite of notable uncontrolled-response mitigation, the peak liquid deflections of the O-TLCD ${ }_{\mu=0.10}$ and the O-TLCD ${ }_{\mu=0.39}$ under the Northridge record in Table 3 indicate that the induced peak liquid deflections $\left(u_{\max }=0.836 \mathrm{~m}\right.$ and $\left.u_{\max }=0.533 \mathrm{~m}\right)$ indeed exceed the provided liquid deflections for $\mathrm{O}-\mathrm{TLCD}_{\mu=0.10}$ and $\mathrm{O}-\mathrm{TLCD}_{\mu=0.39}\left(u_{\text {allwd }}=0.720 \mathrm{~m}, u_{\text {allwd }}=0.450 \mathrm{~m}\right)$. Similar results are observed when we study the peak liquid deflections of other O-TLCDs with 20 and $30 \%$ mass ratios in Table 3 compared with the provided ones $u_{\text {allwd }}$. Therefore, this observation dictates the need for a higher head loss coefficient for the O-TLCDs if they are to be also effective under intenser excitations such as Northridge.

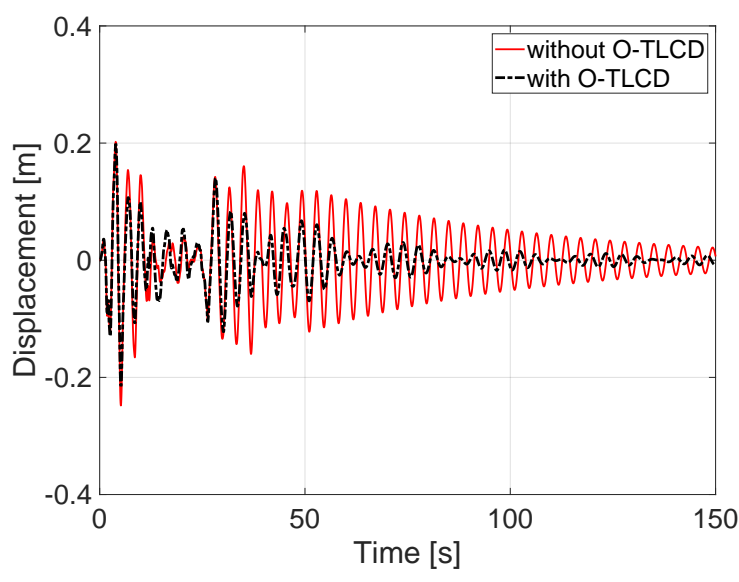

(a) El Centro (1940)

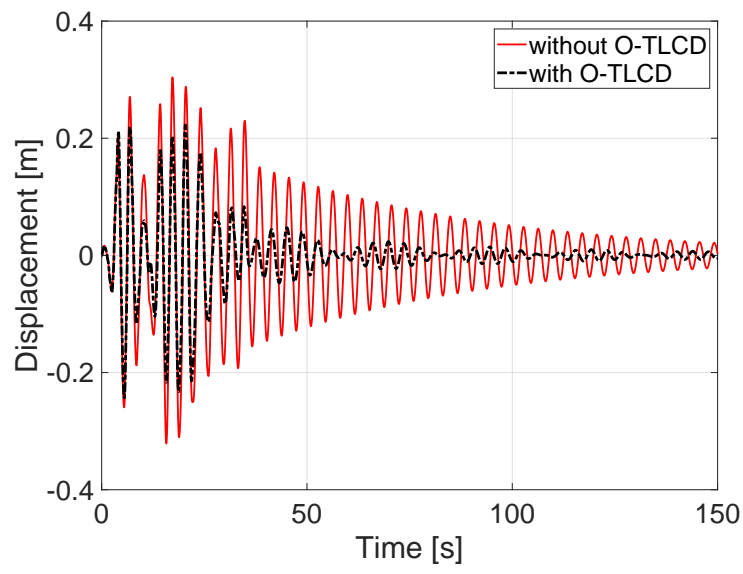

(c) Hachinohe (1964)

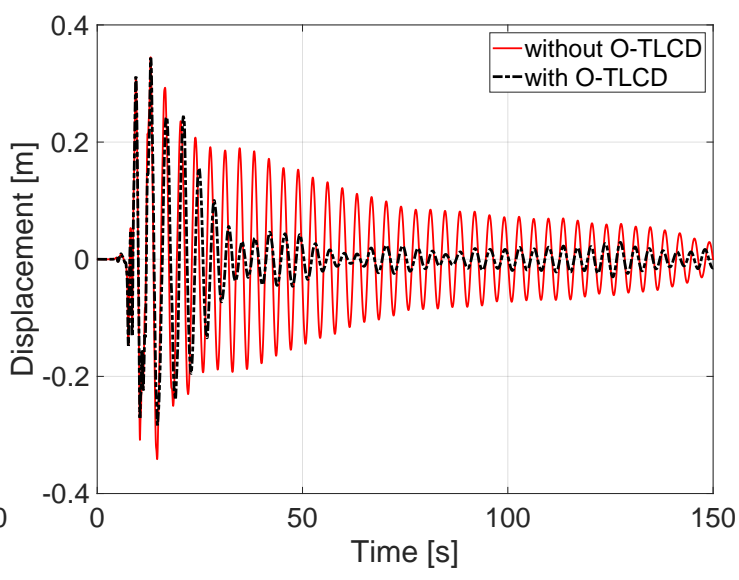

(b) Kobe (1995)

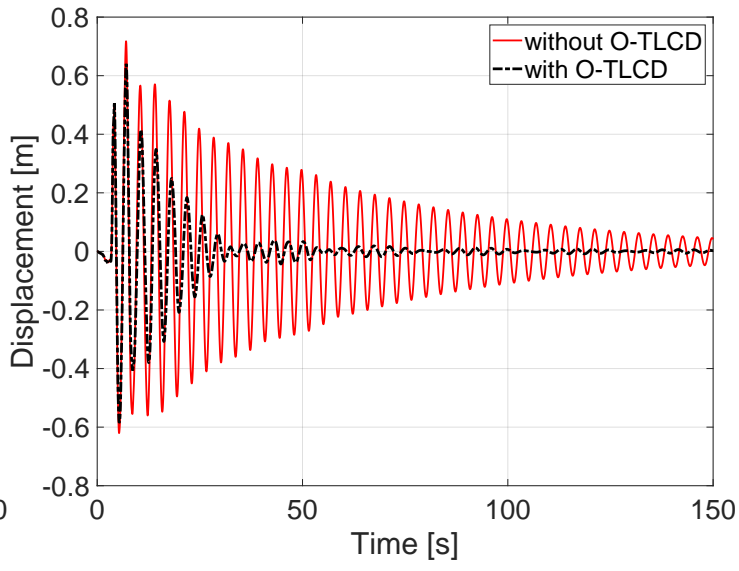

(d) Northridge (1994)

Figure 10: Displacement response of the uncontrolled structure versus the controlled structure equipped with O$\operatorname{TLCD}_{\mu=0.10}$ when $\delta=0.407 \mathrm{~m}^{-1}$ coming from the optimization process under the selected earthquake Kobe. 


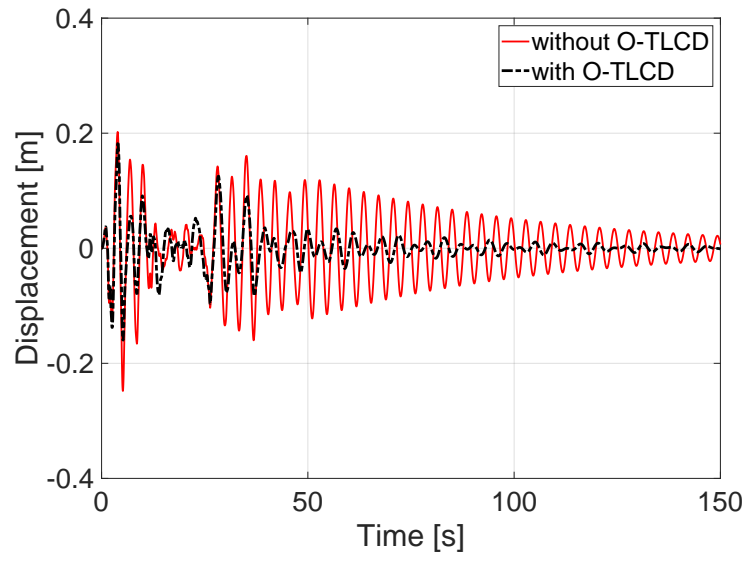

(a) El Centro (1940)

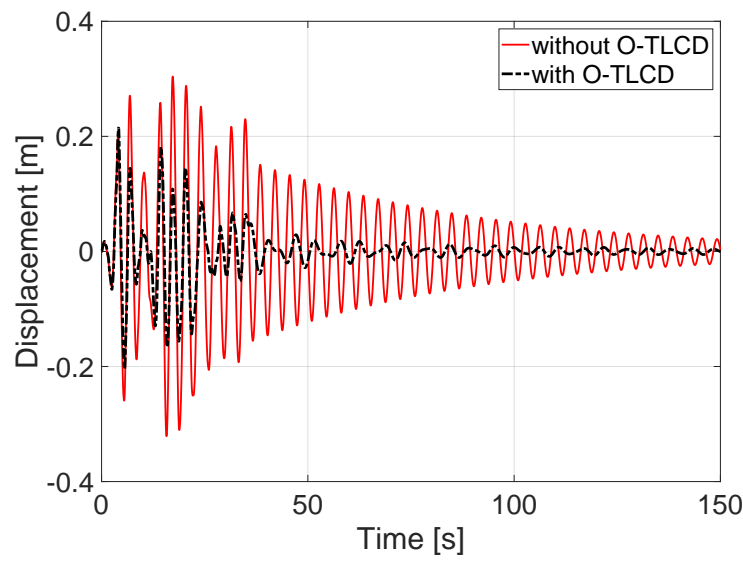

(c) Hachinohe (1964)

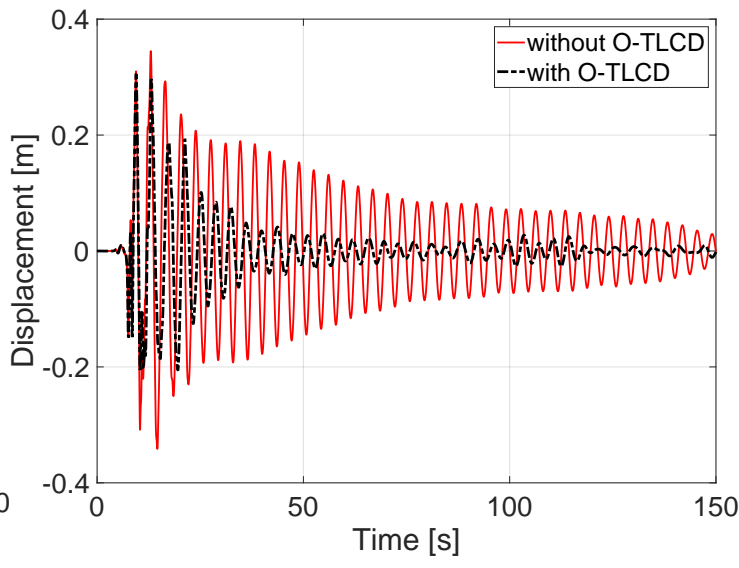

(b) Kobe (1995)

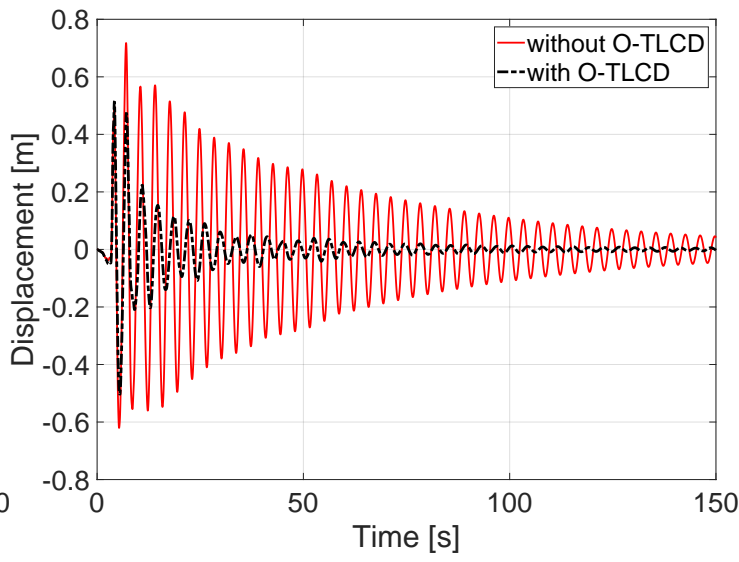

(d) Northridge (1994)

Figure 11: Displacement response of the uncontrolled structure versus the controlled structure equipped with O$\mathrm{TLCD}_{\mu=0.39}$ when $\delta=0.838 \mathrm{~m}^{-1}$ coming from the optimization process under the selected earthquake Kobe.

\subsection{Earthquake excitation, with $\delta_{\text {req }}$}

The higher required head loss coefficient is denoted here by $\delta_{r e q}$. To obtain $\delta_{r e q}$, for each mass ratio, the O-TLCD-structure system is simulated under the Northridge earthquake in a loop where the head loss coefficient of the O-TLCD increases incrementally as long as the induced liquid deflection is greater than the provided one, $u_{\text {allwd }}$. Consequently, the obtained value for the $\delta_{r e q}$ out of each loop for each O-TLCD is reported in Fig. 9. Next, one important aspect is if these values as the head loss coefficient are realistic, for which Eq. 19 and Eq. 20 can be used. For example, for $\mathrm{O}-\mathrm{TLCD}_{\mu=0.39}$, the loss factor $\lambda$ is obtained through the effective liquid length $L=2 \times 0.97+10.34=12.29 \mathrm{~m}$ and $\delta_{r e q}=1.310 \mathrm{~m}^{-1}$ :

$$
\lambda=\delta L=1.310 \times 12.29=16.10,
$$

which is plausible, for instance, by the means of butterfly valves [59] in the midway of the liquid passage in the horizontal columns of the O-TLCD.

Since these required head loss coefficients are to be passively assigned to the corresponding O-TLCDs, all the following assessments are conducted considering that the four O-TLCDs are 
equipped not with the Kobe-optimized head loss coefficient $\delta$ but with the Northridge-required head loss coefficient $\delta_{\text {req }}$. To do this, $\delta$ in Eq. 18 (or Eq. 30) is replaced with $\delta_{\text {req }}$.

It is noted that assigning $\delta_{\text {req }}$, which is generally greater than $\delta$ and therefore limits liquid movements more, influences the O-TLCD performance - can be either improvement or deterioration depending on the vibration content-under less demanding excitations, but important is it can preserve the functionality of the O-TLCD exposed to severer excitations. The peak liquid motion of all four O-TLCDs functioning with their corresponding $\delta$ and $\delta_{r e q}$ under Northridge record are brought in Table 3 where now all the peak liquid deflections using $\delta_{r e q}$ are in the range of $u_{\text {allwd }}$. Subsequently, to evaluate the approximated damping ratio of the O-TLCDs, $D_{d, l i n}$ in Table $2, U_{0}$ and $\delta$ in Eq. 39 are replaced with $u_{\text {allwd }}$ and $\delta_{\text {req }}$, respectively.

For assessment, applying the four earthquake records, Fig. 12 to Fig. 17 present the overall performance of the $\mathrm{O}-\mathrm{TLCD}_{\mu=0.10}$ and the $\mathrm{O}-\mathrm{TLCD}_{\mu=0.39}$ with $\delta_{r e q}$ by plots of the displacement response of the primary structure in the time and the frequency domain as well as the corresponding liquid deflection $u$ from Eq. 30. To achieve a finer resolution in the frequency domain, the simulations are conducted for $2000 \mathrm{sec}$; corresponding sampling rate according to the sample time of each utilized earthquake is employed. For the sake of comparison, the performance of O-TLCDs for all four mass ratios ranging from 10 to $39 \%$ are summarized based on the evaluation of the RMS and the peak of controlled and uncontrolled displacement response of the structure in Fig. 18 with absolute values and in Fig. 19 with relative values with respect to the uncontrolled responses. Here, the uncontrolled responses refer to the structure without O-TLCD (bare structure).

Table 3: Peak amplitude of the liquid deflection $u$ (Eq. 30 of all four O-TLCDs mounted on the structure vibrated under Northridge earthquake with head loss coefficient $\delta$ or $\delta_{\text {req }}$ shown in Fig. 9 versus the allowed deflection $u_{\text {allwd }}$.

\begin{tabular}{ccccc}
\hline & $\mu=0.10$ & $\mu=0.20$ & $\mu=0.30$ & $\mu=0.39$ \\
\hline Allowed liquid deflection $u_{\text {allwd }}[\mathrm{m}]$ & 0.720 & 0.590 & 0.530 & 0.450 \\
Peak liquid deflection with $\delta[\mathrm{m}]$ & 0.836 & 0.610 & 0.601 & 0.533 \\
Peak liquid deflection with $\delta_{\text {req }}[\mathrm{m}]$ & 0.722 & 0.588 & 0.525 & 0.450 \\
\hline
\end{tabular}

It is seen in these two figures that the $\mathrm{O}-\mathrm{TLCD}_{\mu=0.39}$ presents the most effective performance where, according to Fig. 18a, the RMS of the displacement drops from around $10 \mathrm{~cm}$ to almost $5 \mathrm{~cm}$ under the Kobe and at most from $19 \mathrm{~cm}$ to $7 \mathrm{~cm}$ under the Northridge earthquake; $4 \mathrm{~cm}$ under the Kobe and $20 \mathrm{~cm}$ under the Northridge are the least and the most absolute amounts of peak displacement attenuation, respectively. The figures report that, even with the lowest mass ratio, the O-TLCD significantly improves the responses of the structure. According to Fig. 18a, the RMS of the displacement of the structure with the smallest damper O-TLCD ${ }_{\mu=0.10}$ is reduced at least by $31 \%$ under the Kobe and at most by $46 \%$ under the Northridge earthquake. The peak displacement, except a slight increase under the Kobe record, is decreased at least by $11 \%$ under the Northridge and at most by $24 \%$ under the Hachinohe earthquake with this O-TLCD. These results indicate that the structure can experience further vibration mitigation when the attached O-TLCD is designed with a higher mass ratio although this improvement from one mass ratio to the other may not be uniform.

\subsection{Free vibration and harmonic excitation, with $\delta_{\text {req }}$}

For further evaluation, the O-TLCD-structure system is investigated under a harmonic excitation for the O-TLCD ${ }_{\mu=0.10}$ and the O-TLCD ${ }_{\mu=0.39}$. For the uncontrolled case (without $O-T L C D$ ), 


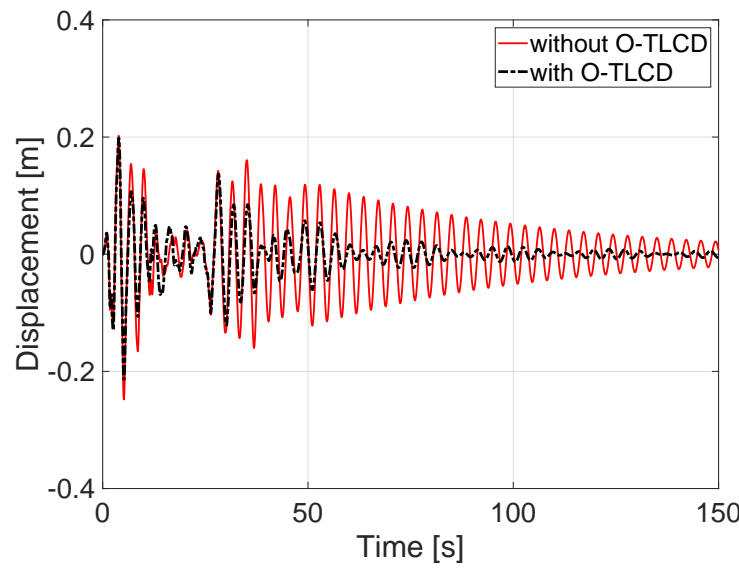

(a) El Centro (1940)

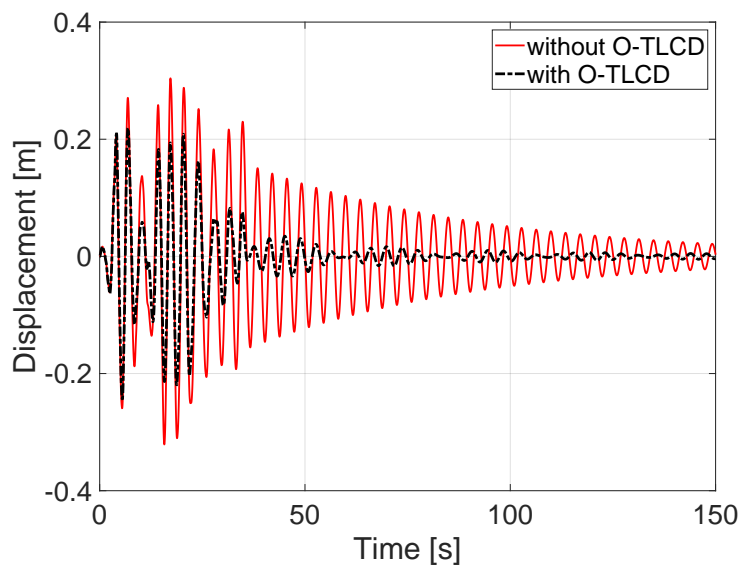

(c) Hachinohe (1964)

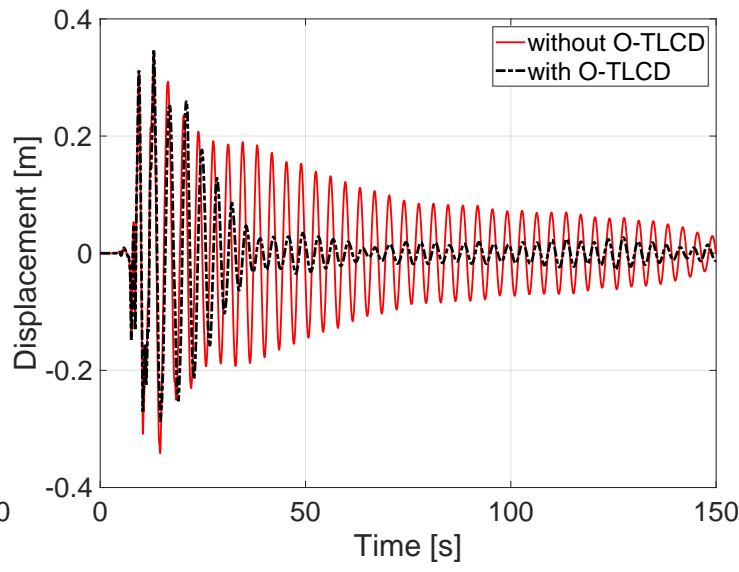

(b) Kobe (1995)

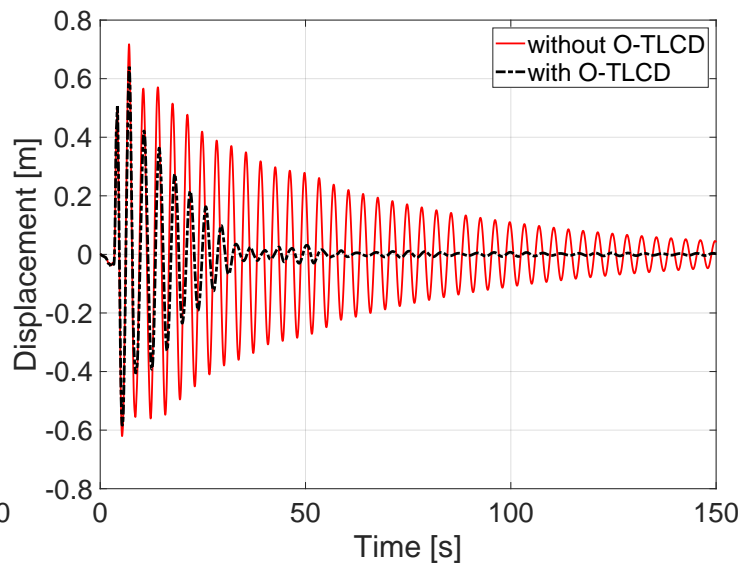

(d) Northridge (1994)

Figure 12: Displacement response of the uncontrolled structure versus the controlled structure equipped with O$\mathrm{TLCD}_{\mu=0.10}$ when $\delta_{r e q}=0.579 \mathrm{~m}^{-1}$ (Northridge-required head loss coefficient) under earthquakes.

it is obvious that the excitation frequency should equal to $\omega_{s}$. To evaluate the resonant frequency of the controlled case (with $O-T L C D$ ), we conduct a free vibration test with initial displacement of $0.15 \mathrm{~m}$ for $2000 \mathrm{~s}$ and process the displacement response using fast Fourier transform (FFT) with sampling rate of $100 \mathrm{~Hz}$, which is brought in Fig. 20 and Fig. 21 in time interval of $300 \mathrm{~s}$ and frequency band of $0.5 \mathrm{~Hz}$ for the $\mathrm{O}-\mathrm{TLCD}_{\mu=0.10}$ and the $\mathrm{O}-\mathrm{TLCD}_{\mu=0.39}$, respectively. In these figures, as mentioned earlier, off $O-T L C D$ refers to the O-TLCD-structure system where the O-TLCD exists but is deactivated.

The response in the frequency domain in Fig. 21b shows that the system with off O-TLCD ${ }_{\mu=0.39}$ presents

$$
f_{s, d}=f_{s} \sqrt{\frac{m_{s}}{m_{s}+m_{d}}}=0.280 \sqrt{\frac{400}{400+155.74}}=0.238 \mathrm{~Hz},
$$

which is the natural frequency of the structure carrying the whole mass of the O-TLCD $\mu=0.39$ with stationary liquid; $f_{s, d}=0.267$ for the O-TLCD ${ }_{\mu=0.10}$. On the other hand, when the O-TLCD is activated (with O-TLCD), $f_{s, d}$ is split into a lower and a higher frequency, which according 


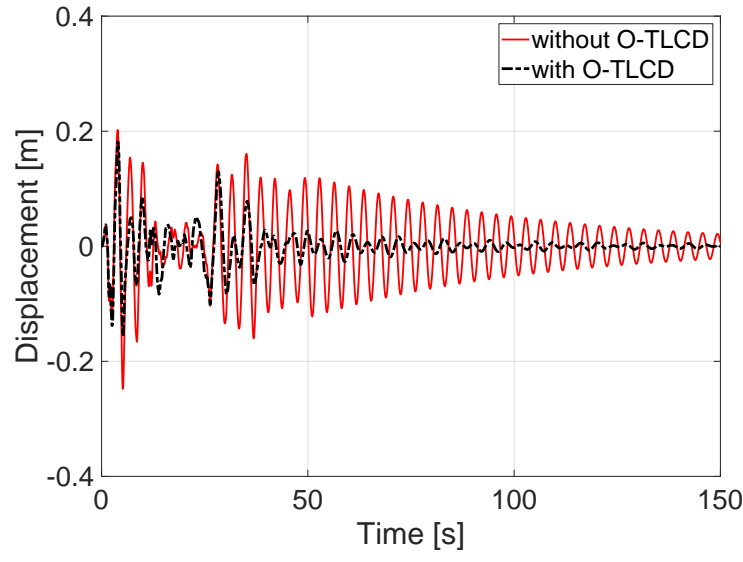

(a) El Centro (1940)

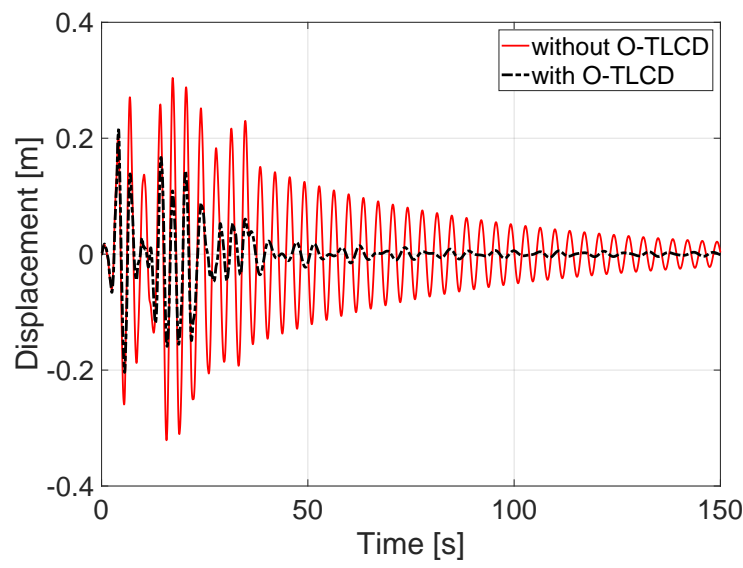

(c) Hachinohe (1964)

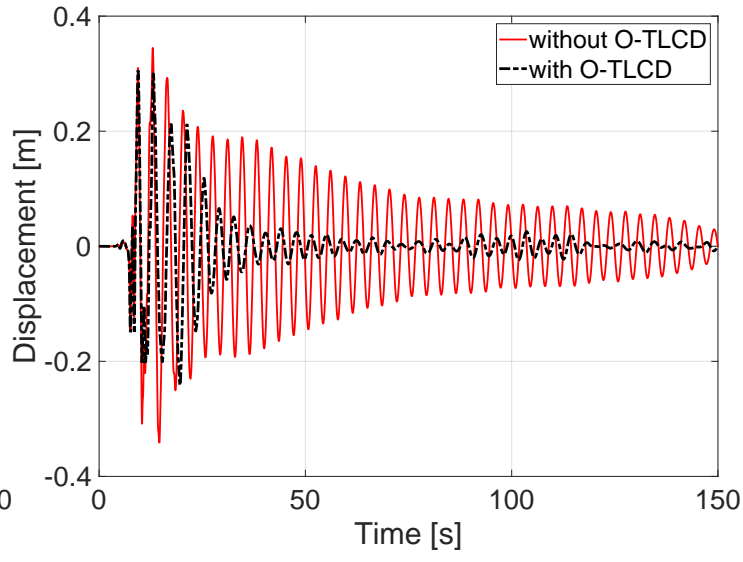

(b) Kobe (1995)

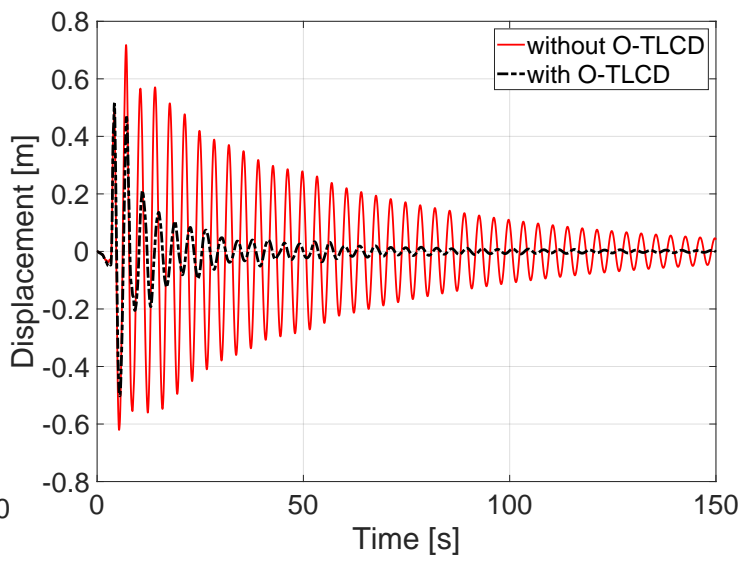

(d) Northridge (1994)

Figure 13: Displacement response of the uncontrolled structure versus the controlled structure equipped with O$\mathrm{TLCD}_{\mu=0.39}$ when $\delta_{r e q}=1.310 \mathrm{~m}^{-1}$ (Northridge-required head loss coefficient) under earthquakes.

to Fig. 21b, are $f_{s, d, l}=0.187 \mathrm{~Hz}$ and $f_{s, d, r}=0.270 \mathrm{~Hz}$, respectively, for the O-TLCD ${ }_{\mu=0.39}$; $f_{s, d, l}=0.243 \mathrm{~Hz}$ and $f_{s, d, r}=0.283 \mathrm{~Hz}$ for the O-TLCD $\mu=0.10$.

Therefore, the harmonic excitation is defined as

$$
\ddot{w}_{g}=A \sin \left(\omega_{\text {res }} t\right),
$$

where, for the O-TLCD ${ }_{\mu=0.39}$, by choosing the right frequency of the coupled system $f_{s, d, r}$,

$$
\omega_{\text {res }}= \begin{cases}\omega_{s}=0.28 \times 2 \pi=1.759 \mathrm{rad} \mathrm{s}^{-1} & \text { for the uncontrolled structure (without O-TLCD) } \\ \omega_{s, d, r}=0.270 \times 2 \pi=1.697 \mathrm{rad} \mathrm{s}^{-1} & \text { for the controlled structure (with O-TLCD) }\end{cases}
$$

and $A$ is an appropriate amplitude (here $A=0.05 \mathrm{~m} \mathrm{~s}^{-2}$ ); $\omega_{s, d, r}=1.778 \mathrm{rad} \mathrm{s}^{-1}$ for the O$\operatorname{TLCD}_{\mu=0.10}$. The displacement response of the coupled system and the corresponding liquid deflection under the harmonic excitation in Eq. 45 are depicted for the O-TLCD $\mu=0.10$ with $\delta_{r e q}=0.579 \mathrm{~m}^{-1}$ and the O-TLCD ${ }_{\mu=0.39}$ with $\delta_{r e q}=1.310 \mathrm{~m}^{-1}$ in Fig. 22 and Fig. 23 , respectively. It is noted, since the employed head loss coefficients $\delta_{r e q}$ are generally higher than the 


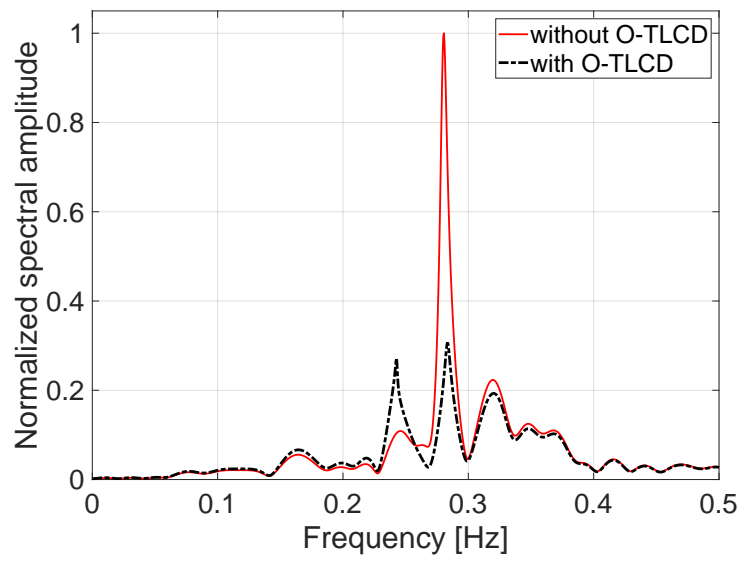

(a) El Centro (1940)

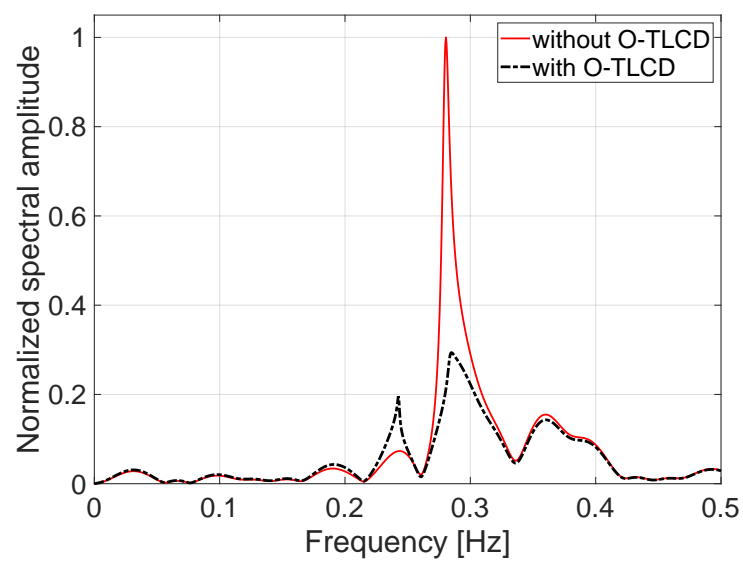

(c) Hachinohe (1964)

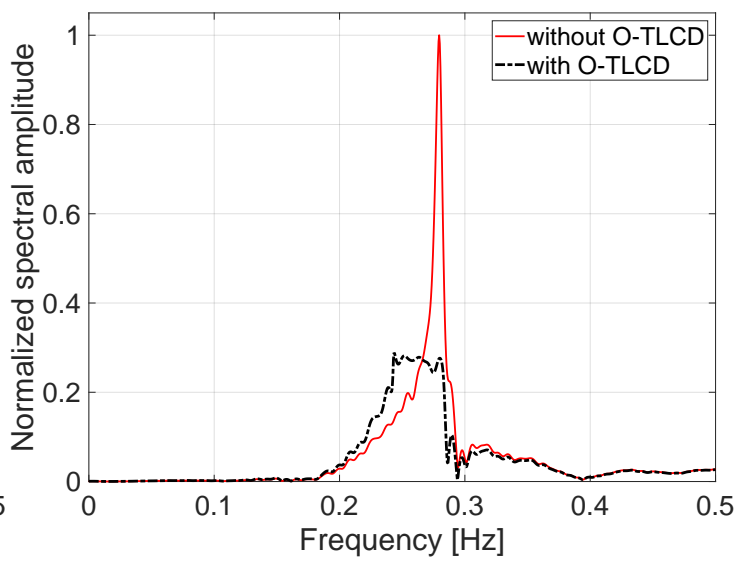

(b) Kobe (1995)

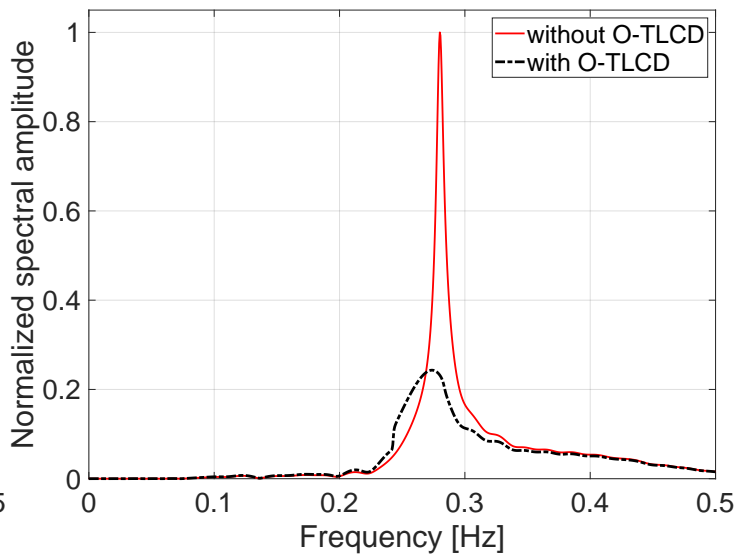

(d) Northridge (1994)

Figure 14: Spectral amplitude (normalized to the peak uncontrolled amplitude) of the response of the uncontrolled structure versus the controlled structure equipped with $\mathrm{O}-\mathrm{TLCD}_{\mu=0.10}$ when $\delta_{r e q}=0.579 \mathrm{~m}^{-1}$ (Northridge-required head loss coefficient) under earthquakes.

obtained optimal one $\delta$ (Table 1), the peak response of the coupled system may occur not at the right but at a slightly lower frequency, which is the result of excessive damping on the optimal tuning (see e.g. [58]) and is neglected here.

It is also worth mentioning that the two points of intersection of with-O-TLCD and off-O$T L C D$ cases in the middle of the spectrum graph in Fig. $21 \mathrm{~b}$ are not in the same level, which may be interpreted as not properly optimized tuning of the dampers, for which the reason can be twofold. First, the utilized tuning formulas (Warburton) have been proposed for undamped structures while the structure of the current study presents slight damping. Second, the free vibration causes harmonic oscillations, for which Den Hartog's tuning criterion,

$$
f_{d}=f_{d, o p t}=\frac{f_{s}^{*}}{1+\mu^{*}}=\frac{0.265}{1+0.247}=0.213 \mathrm{~Hz},
$$

for a theoretic O-TLCD ${ }_{\mu=0.39}$ (here we neglect that the layout of a real O-TLCD affects $f_{s}^{*}$ and $\mu^{*}$, too) with the same $\delta_{r e q}=1.310 \mathrm{~m}^{-1}$ results in the performance shown in Fig. 24d, which brings the two points of intersection on the $y$ axis closer to each other. Similar influence is seen for the 


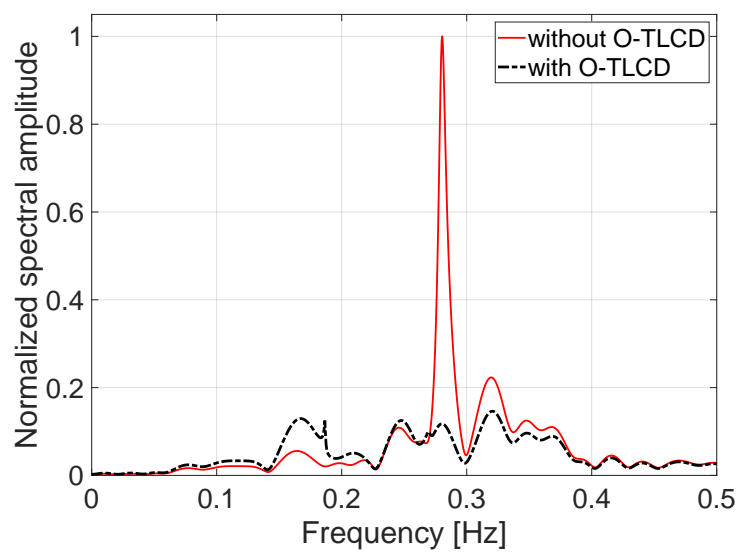

(a) El Centro (1940)

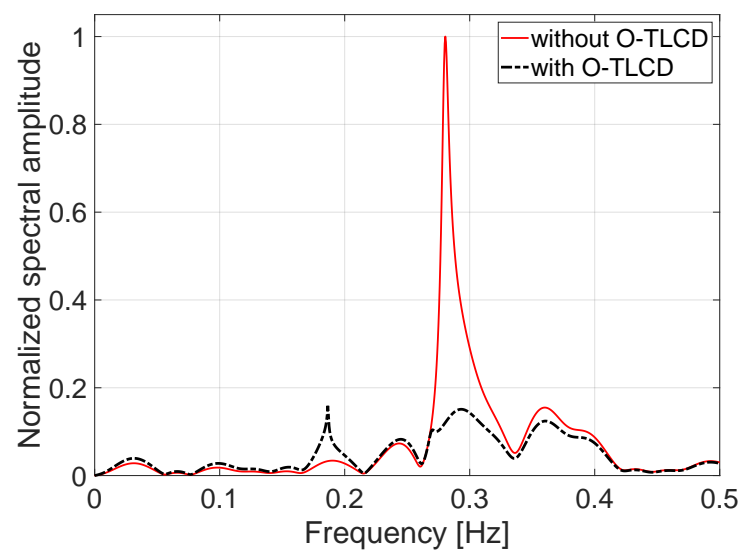

(c) Hachinohe (1964)

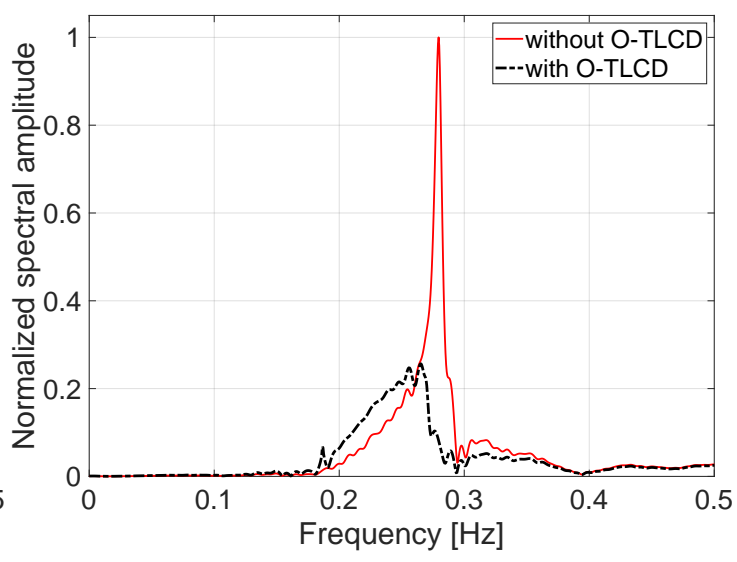

(b) Kobe (1995)

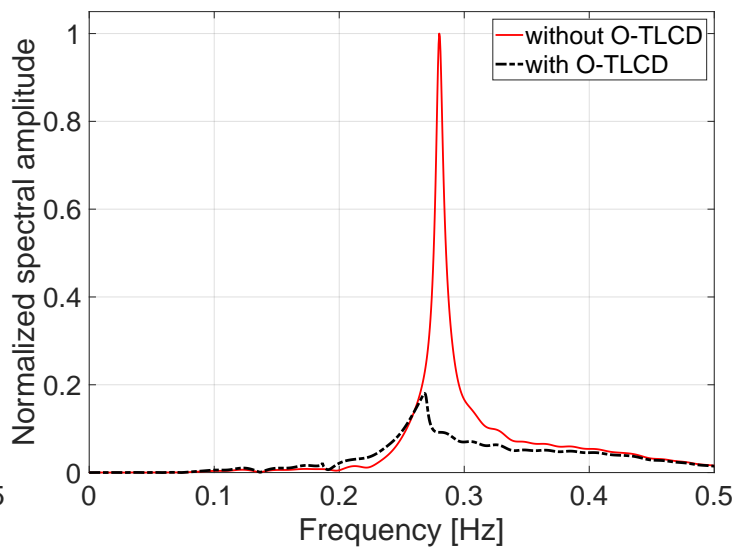

(d) Northridge (1994)

Figure 15: Spectral amplitude (normalized to the peak uncontrolled amplitude) of the response of the uncontrolled structure versus the controlled structure equipped with $\mathrm{O}-\mathrm{TLCD}_{\mu=0.39}$ when $\delta_{r e q}=1.310 \mathrm{~m}^{-1}$ (Northridge-required head loss coefficient) under earthquakes.

other mass ratios in this figure.

This observation, however, cannot necessarily lead to a better performance for a random vibration, because of which the Warburton criteria were chosen for this study although the investigations of the authors (not reported here) on different types of vibrations using different damper frequencies highlight that the Warburton's criteria cannot be taken as impeccable tuning principles for a whole vibration scenario either. This issue, like the discussion of the required head loss coefficient mentioned earlier, remarks that there would be a need for a continuous tunability in liquid dampers, in which the O-TLCD is no exception, to realize their full potential. Moreover, Fig. 24 reports that the more the mass ratio increases the wider frequency band is used to split the frequency of the off-O-TLCD-structure system.

\subsection{White-noise excitation, with $\delta_{\text {req }}$}

The last contribution of this study in the numerical investigation part is the application of a random-generated signal as the excitation. In doing so, the performance of the lightest and the heaviest O-TLCDs have been investigated under band-limited white noise, with sample time of 


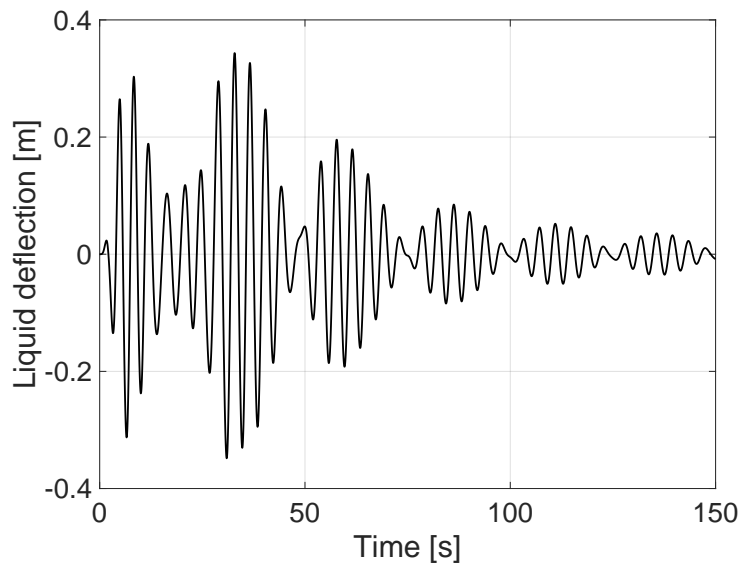

(a) El Centro (1940)

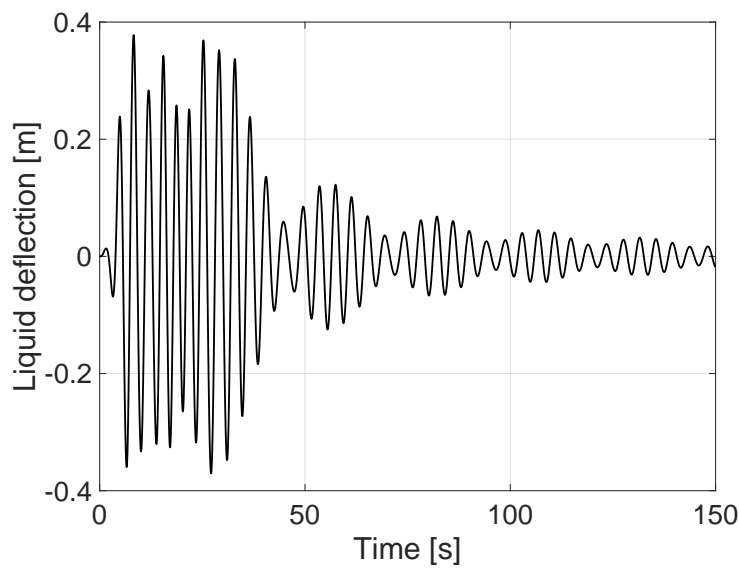

(c) Hachinohe (1964)

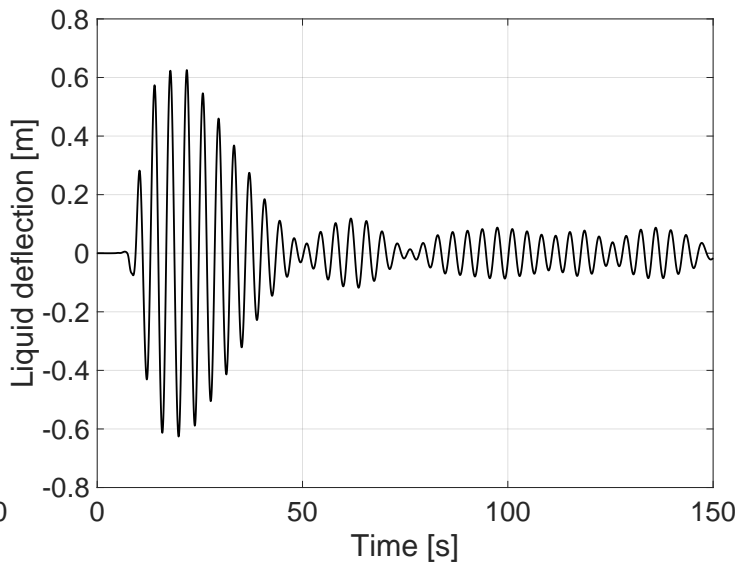

(b) Kobe (1995)

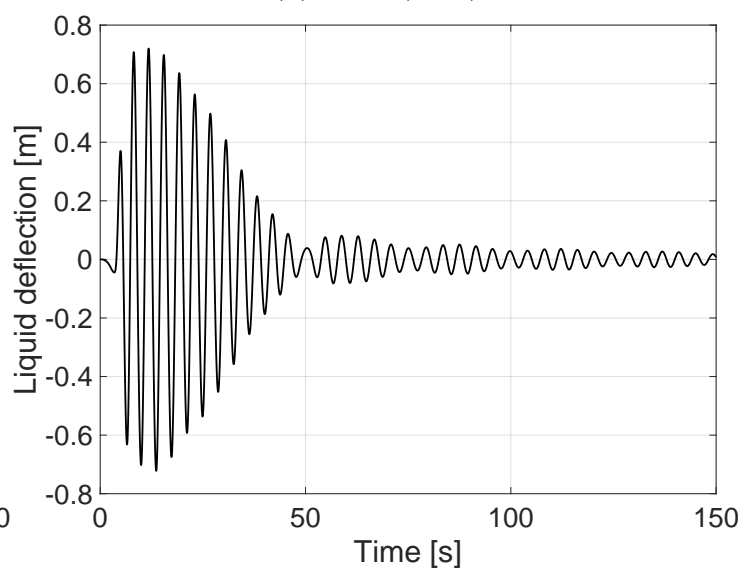

(d) Northridge (1994)

Figure 16: Time-history plots of liquid deflection $u$ (from Eq. 30 of O-TLCD ${ }_{\mu=0.10}$ with $\delta_{r e q}=0.579 \mathrm{~m}^{-1}$ on the structure excited by earthquakes.

$0.01 \mathrm{~s}$ and noise power of 0.005 , applied to the structure as a base acceleration lasting for $300 \mathrm{~s}$ in the Simulink/Matlab. Results in the time and the frequency domain brought in Fig. 25 once again confirm here that even the $\mathrm{O}-\mathrm{TLCD}_{\mu=0.10}$ has been able to reduce excessive vibrations of the structure acceptably. On the other hand, and similar to the case under the real earthquake records, $\mathrm{O}-\mathrm{TLCD}_{\mu=0.39}$ performs the highest response reductions with the least liquid movement.

Chief among these observations is that this effective performance of the O-TLCD is not excitationdirection dependent, but omnidirectional. In other words, regardless of the excitation angle of incidence, the O-TLCD provides the structure with the same control force all along a vibration scenario. This omnidirectionality, without adding extra complication coming from extra DoFs to the motion equation of the damper, is accounted as a remarkable advantage compared to other counterparts such as TLCDs whose control forces fade when the excitation diverges from the damper's alignment. 


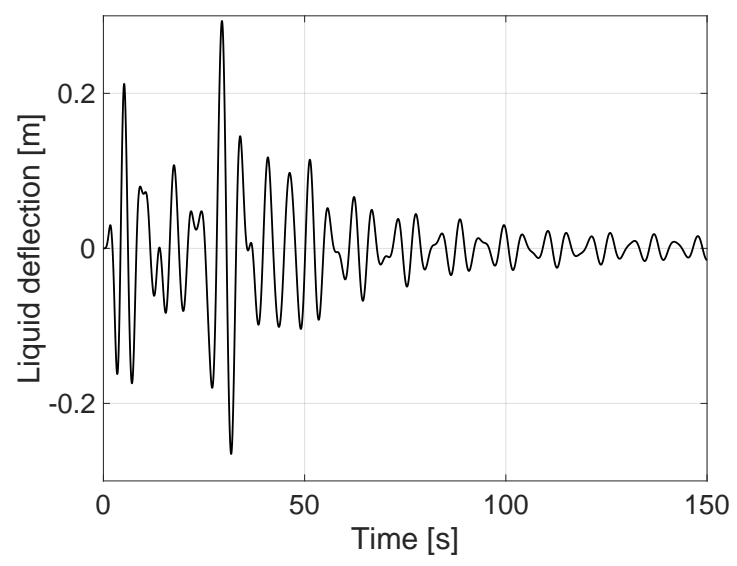

(a) El Centro (1940)

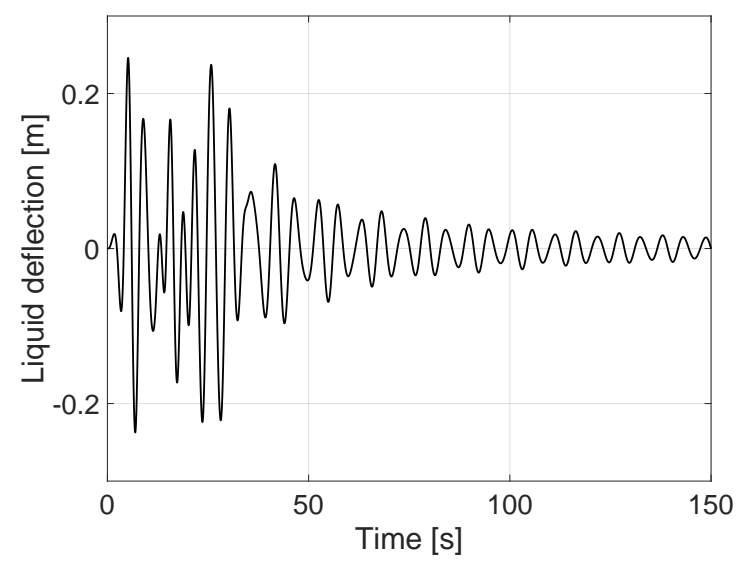

(c) Hachinohe (1964)

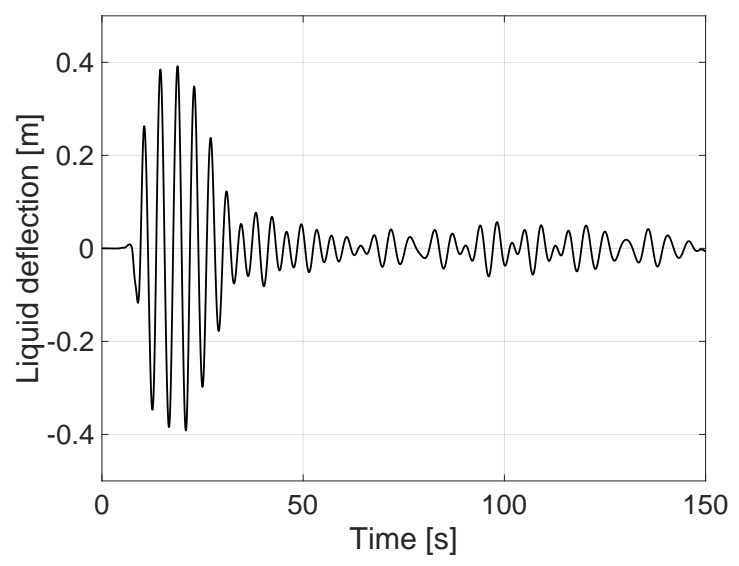

(b) Kobe (1995)

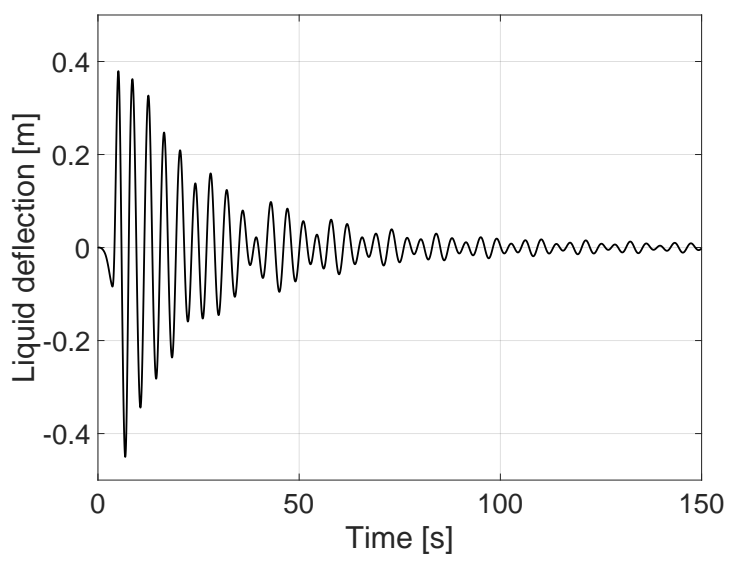

(d) Northridge (1994)

Figure 17: Time-history plots of liquid deflection $u$ (from Eq. 30 of O-TLCD ${ }_{\mu=0.39}$ with $\delta_{\text {req }}=1.310 \mathrm{~m}^{-1}$ on the structure excited by earthquakes.

\section{Experimental Investigations}

This section investigates the validity of the proposed mathematical representation of the OTLCDs experimentally. For this purpose, an O-TLCD prototype is fabricated and experiments on it are conducted at the Control Engineering Laboratory of the RWTH Aachen University. The focus of the experiments is two governing equations: (i) calculation of the natural frequency of the O-TLCDs (Eq. 19) and (ii) calculation of the response of the O-TLCDs subjected to different excitation angle of incidence (Eq. 18).

\subsection{Description of the experimental setup}

The O-TLCD prototype is fabricated using transparent PVC-U pipes (inner diameter $=55 \mathrm{~mm}$ ) connected to each other by PVC-U angles $90^{\circ}$ and a PVC-U crosspiece with adhesive sleeves (inner diameter $=63 \mathrm{~mm}$ ) as shown in Fig. 26. This O-TLCD has four L-arms (see numbers 1 to 4 ) with equal vertical and horizontal cross sections distributed symmetrically around the center, where the liquid level in the vertical columns is $V=121 \mathrm{~mm}$ and the horizontal length of each L-arm is $H / 2=270 \mathrm{~mm}$. Furthermore, in order to provide a reference device, a classical TLCD with 


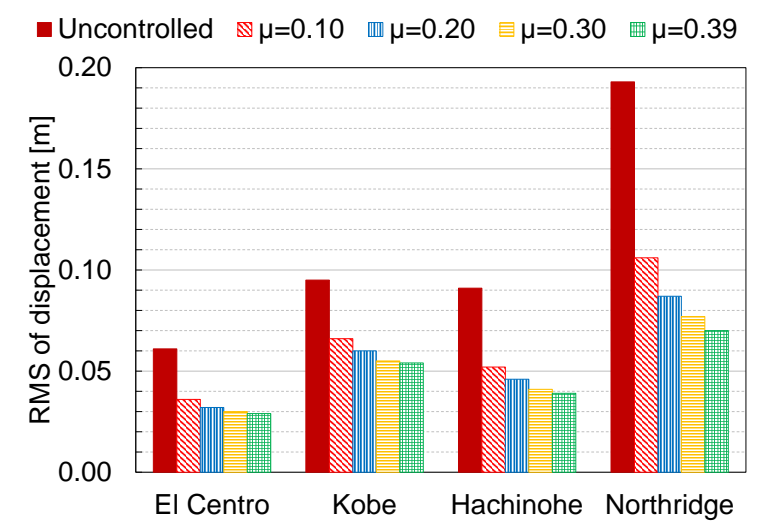

(a)

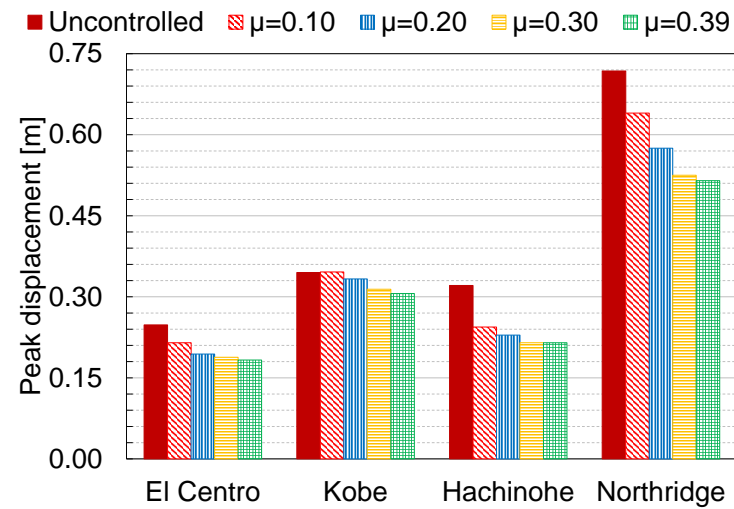

(b)

Figure 18: Displacement absolute values: the RMS (a) and the peak (b) response evaluation of the O-TLCD-structure system (controlled) with different mass ratios $\mu$ and with the corresponding $\delta_{r e q}$ (Fig. 9) versus the response of the bare (uncontrolled) structure under four earthquakes.

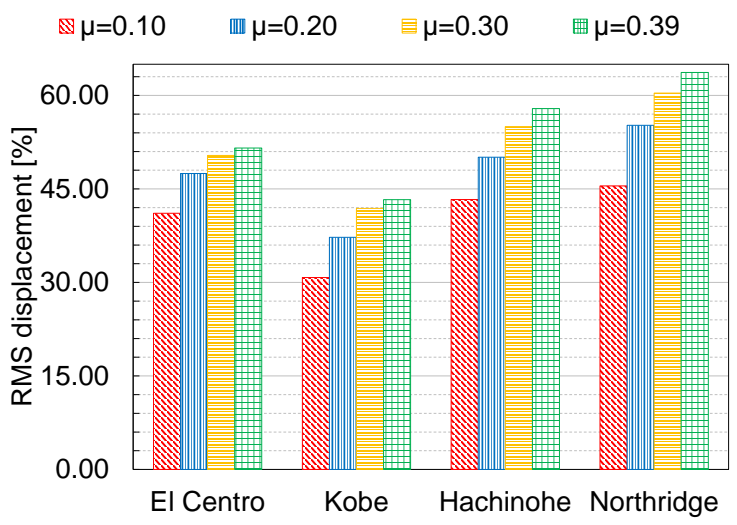

(a)

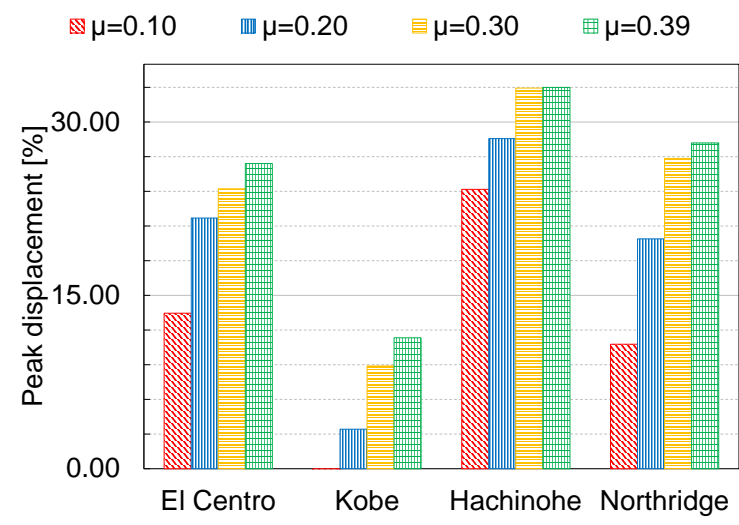

(b)

Figure 19: Displacement reduction percentage: the RMS (a) and the peak (b) response evaluation of the O-TLCDstructure system (controlled) with different mass ratios $\mu$ and with the corresponding $\delta_{r e q}$ (Fig. 9) relative to the response of bare (uncontrolled) structure under four earthquakes.

two vertical columns is also fabricated using the same material with the same dimensions of inner diameter, $V$ and $H$.

As shown in Fig. 26, the O-TLCD (as well as the TLCD) prototype is tested on a uniaxial shaking table (5). The shaking table is built in a way that a threaded rod, which is operated by a step motor with the frequency range of $0.10-50 \mathrm{~Hz}$ and $\pm 50 \mathrm{~mm}$ stroke, forms the horizontal motion of the O-TLCD. The O-TLCD is mounted on the shaking table using two rectangular wooden platforms $(6$ and 7$)$. The lower platform $(6)$ is directly fixed to the shaking table; the upper one (7) is fixed to the O-TLCD and can be screwed to the lower platform at two diagonal points $(8)$. At these two points, two steel threaded inserts are placed on the lower platform, which guarantee no relative movement between the platforms. Accordingly, on the diagonal quarters of the upper platform, seven holes (orienting holes) are equally spaced at $15^{\circ}$ in a circular pattern 


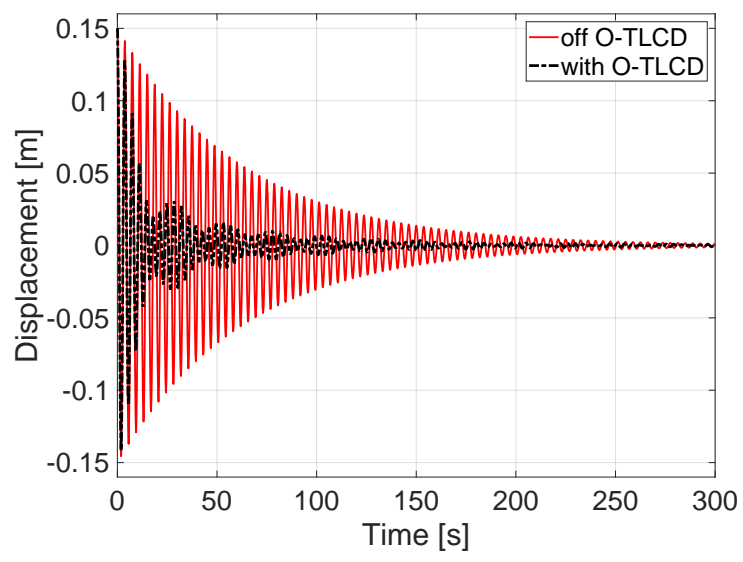

(a)

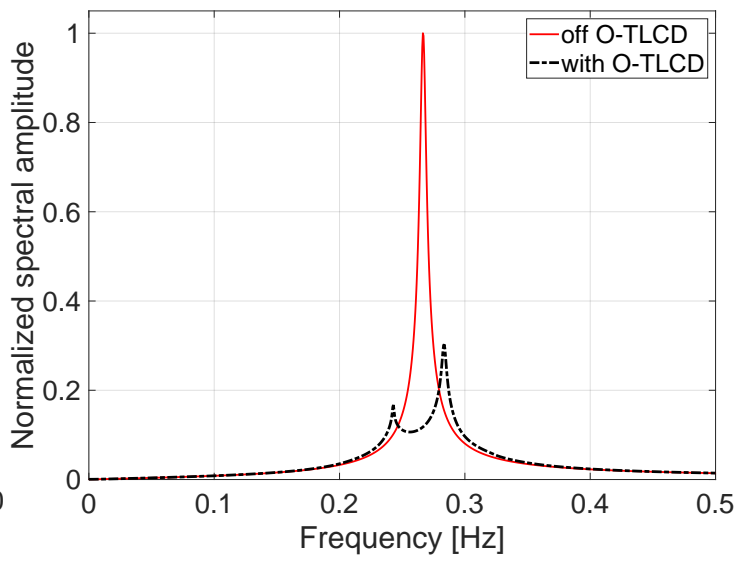

(b)

Figure 20: Free vibration: Displacement response of the $\mathrm{O}-\mathrm{TLCD}_{\mu=0.10}$-structure system for two cases of the deactivated (off O-TLCD) and the activated (with O-TLCD) O-TLCD with $\delta_{r e q}=0.579 \mathrm{~m}^{-1}$ in the time domain (a) and the frequency domain (b) when $0.15 \mathrm{~m}$ initial displacement is applied to the structure.

so that the upper platform fixed to the O-TLCD can be oriented in seven different ways ranging from 0 to $90^{\circ}$ with respect to the motion direction of the shaking table (Fig. 26a). With this setup multidirectional vibration tests are conducted on the O-TLCD.

The liquid motion is dynamically measured using an ultrasonic distance measuring sensor $(9)$, UNDK 30U6112/S14, which is installed over the vertical column of the L-arm 1. Furthermore, the motion of the shaking table is independently monitored by a laser sensor (10), by which the accurate application of the requested excitation is validated. The data acquisition is conducted by a micro-controller with an analog-to-digital (A/D) converter and processed in Simulink/Matlab. The Simulink model is compiled in a real-time PC and the control command is sent to the step motor. In this setup, the measurements are recorded with the sample rate of $100 \mathrm{~Hz}$. For the sake of statistical accuracy, each test is conducted at least three times and the average values are reported.

\subsection{Natural frequency of the $O-T L C D$}

The natural frequencies of the O-TLCD prototype are determined experimentally by frequency sweeps. The O-TLCD is positioned at $\alpha=0^{\circ}$ (L-arm 1 is aligned with the motion of the shaking table as shown in Fig. 26p and a series of tests are conducted under harmonic excitation of the form,

$$
w=A \sin (2 \pi f t),
$$

where the excitation amplitude $A$ is a constant equal to $4.5 \mathrm{~mm}$ and the excitation frequency $f$ ranges from $0.65 \mathrm{~Hz}$ to $1.05 \mathrm{~Hz}$ with an increment of $0.02 \mathrm{~Hz}$. For the sake of accuracy, the frequency interval is divided by a finer increment of $0.005 \mathrm{~Hz}$ about the expected resonance region. The frequency sweep test is performed for the TLCD prototype as well.

From the measured liquid deflection $u_{1}$ (the liquid deflection in L-arm 1) the response curves of both prototypes are determined and illustrated in Fig. 27. The frequencies of the peak values of the response curves correspond to the natural frequencies of the tested prototypes. Using Eq. 19 , the analytical natural frequencies of both devices are calculated by substituting $V=121 \mathrm{~mm}$ and $H / 2=270 \mathrm{~mm}$. The experimental and analytical results are gathered and compared in Table 4 . 


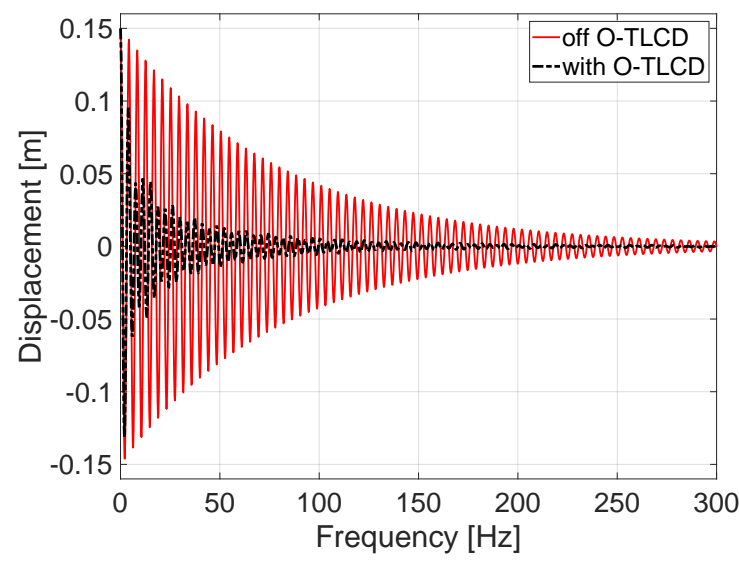

(a)

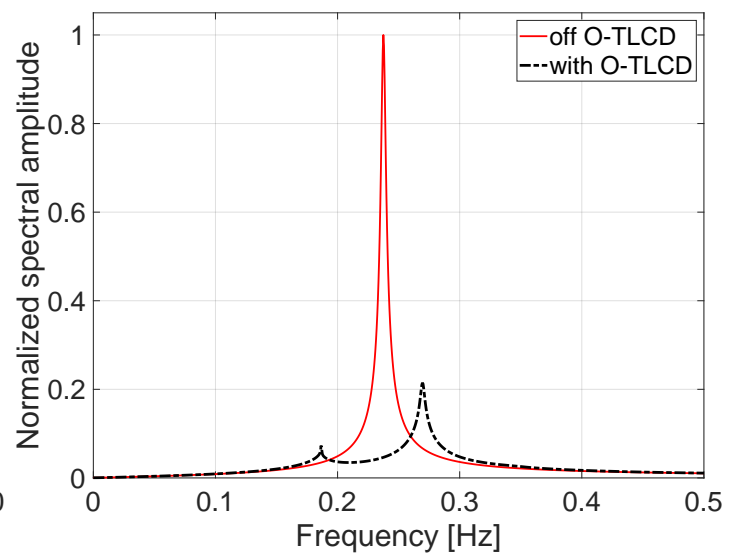

(b)

Figure 21: Free vibration: Displacement response of the $\mathrm{O}-\mathrm{TLCD}_{\mu=0.39}$-structure system for two cases of the deactivated (off O-TLCD) and the activated (with O-TLCD) O-TLCD with $\delta_{r e q}=1.310 \mathrm{~m}^{-1}$ in the time domain (a) and the frequency domain (b) when $0.15 \mathrm{~m}$ initial displacement is applied to the structure.

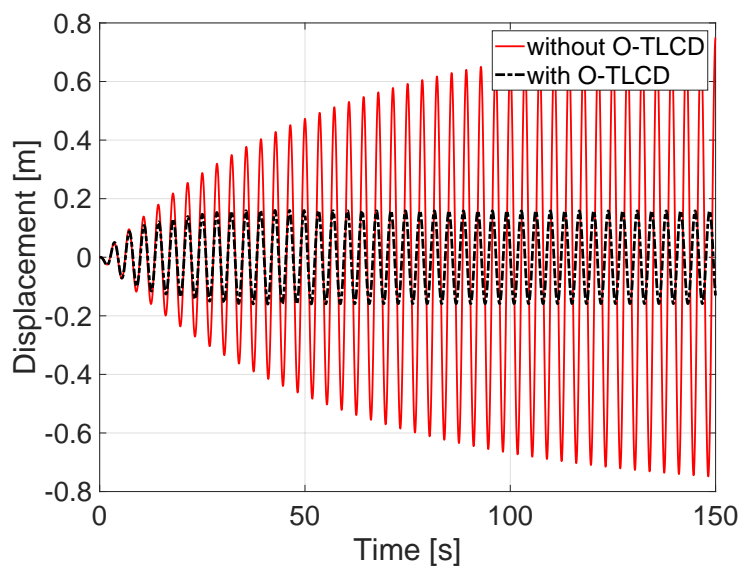

(a)

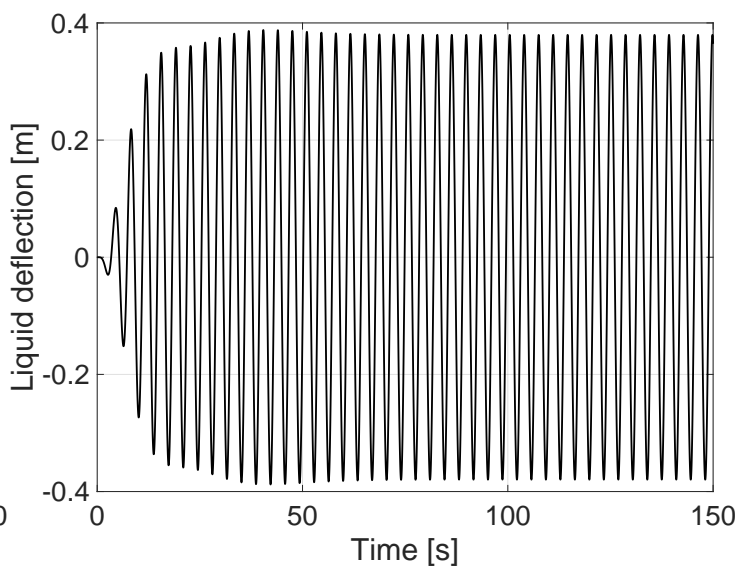

(b)

Figure 22: Harmonic excitation: Displacement response of the uncontrolled (bare) structure versus the controlled structure equipped with $\mathrm{O}-\mathrm{TLCD} \mathrm{C}_{\mu=0.10}$ with $\delta_{\text {req }}=0.579 \mathrm{~m}^{-1}$ under the base acceleration $0.05 \sin \left(\omega_{\text {res }} t\right)$ where $1.759 \mathrm{rad} \mathrm{s}^{-1}$ for the uncontrolled and $1.778 \mathrm{rad} \mathrm{s}^{-1}$ for the controlled structure are considered as $\omega_{\text {res }}$ (a) and the corresponding liquid deflection $u$ from Eq. 30 (b).

Table 4: Natural frequencies of the O-TLCD and TLCD prototypes determined experimentally from the frequency sweep tests and calculated by Eq. 19

\begin{tabular}{lccc}
\hline & $\begin{array}{c}\text { Experiment } \\
f_{\exp }[\mathrm{Hz}]\end{array}$ & $\begin{array}{c}\text { Calculation } \\
f_{\text {calc }}[\mathrm{Hz}]\end{array}$ & $\begin{array}{c}\text { Difference } \\
\Delta f[\mathrm{~Hz}]\end{array}$ \\
\hline O-TLCD & 0.830 & 0.797 & 0.033 \\
TLCD & 0.825 & 0.797 & 0.025 \\
\hline
\end{tabular}

The obtained experimental natural frequencies are both for the O-TLCD and TLCD prototypes very close to the calculation results. The frequency difference is acceptable and can be justified 


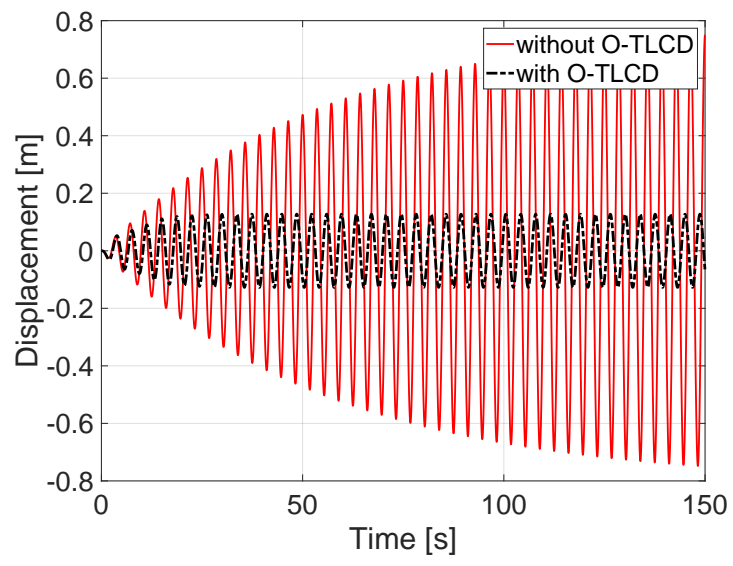

(a)

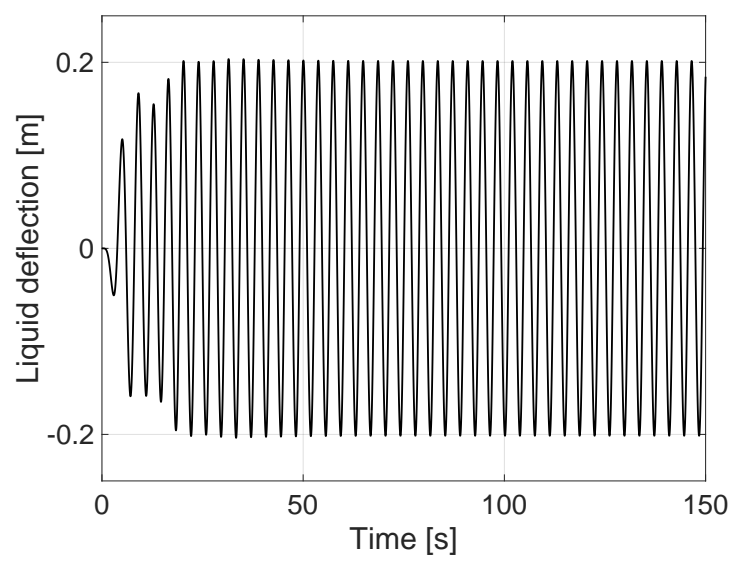

(b)

Figure 23: Harmonic excitation: Displacement response of the uncontrolled (bare) structure versus the controlled structure equipped with O-TLCD ${ }_{\mu=0.39}$ with $\delta_{\text {req }}=1.310 \mathrm{~m}^{-1}$ under the base acceleration $0.05 \sin \left(\omega_{\text {res }} t\right)$ where $1.759 \mathrm{rad} \mathrm{s}^{-1}$ for the uncontrolled and $1.696 \mathrm{rad} \mathrm{s}^{-1}$ for the controlled structure are considered as $\omega_{\text {res }}$ (a) and the corresponding liquid deflection $u$ from Eq. 30 (b).

with the uncertainties regarding the shaking table response and the frequency resolution of the measured data. Furthermore, the calculations are based on the simplified dimensions $V$ and $H / 2$, which do not consider the exact geometries of the prototypes and the liquid flow.

\subsection{Response of the $O-T L C D$ under different excitation angle}

To evaluate the response calculation of O-TLCDs, the aforementioned experimental tests are conducted numerically. The main purpose of this investigation is to verify the equation of motion of the O-TLCD (Eq. 18) considering particularly the representation of the effects of the excitation direction. The core of the presented equation of motion in Eq. 18 is the proposed definition of the DoF of the O-TLCDs in Eq. 1, which assumes a pseudo TLCD in the excitation direction and relates the liquid motion of this TLCD, $u$, to the liquid motions in the tubes of the O-TLCD, $u_{i}$.

In the first part of the investigation, the frequency sweep tests are simulated for both OTLCD and TLCD prototypes under the harmonic excitation given in Eq. 48 in Simulink/Matlab. The resulted two spectra are added to Fig. 27. Here, the natural frequencies and the head loss coefficients of both devices are tuned to the resonance case. Accordingly, we simulate the peak value $u_{1, \max }=42.2 \mathrm{~mm}$ for O-TLCD with $f_{d}=0.830 \mathrm{~Hz}$ and $\delta=2.19 \cdot 10^{-3} \mathrm{~mm}^{-1}$. For TLCD, we use $f_{d}=0.825 \mathrm{~Hz}$ and $\delta=2.26 \cdot 10^{-3} \mathrm{~mm}^{-1}$. Wider frequency response spectra of the simulation shows that the nonlinear damping representation used in the mathematical models basically predicts more inherent damping than the one the prototypes presented physically in the laboratory.

In the second part of the investigation, the free vibration response of the O-TLCD is studied by applying on the damper an impulse excitation by the shaking table. At each test, the orientation of the O-TLCD, $\alpha$, is varied from 0 to $45^{\circ}$ with the increment of $15^{\circ}$ with respect to the motion direction of the shaking table. The liquid motions for each excitation direction are recorded in the L-arm 1 for about $80 \mathrm{~s}$ and after zero padding till $1000 \mathrm{~s}$ are transformed into the frequency domain by the FFT. The results are shown in Fig. 28 which confirms that the natural frequency of the O-TLCD remains invariable by changing the excitation direction. 


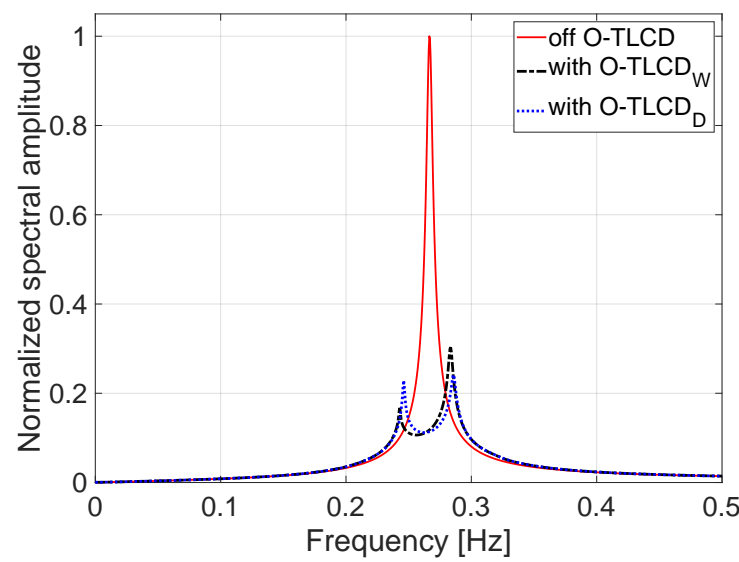

(a) $\mu=0.10$

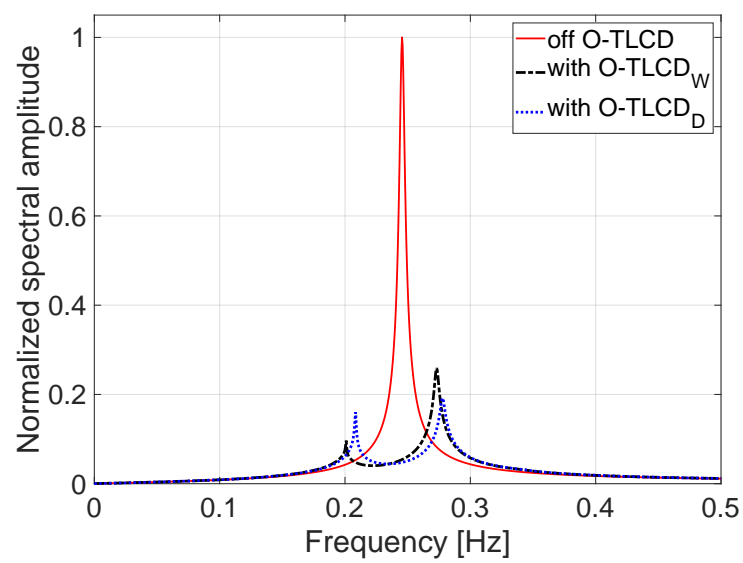

(c) $\mu=0.30$

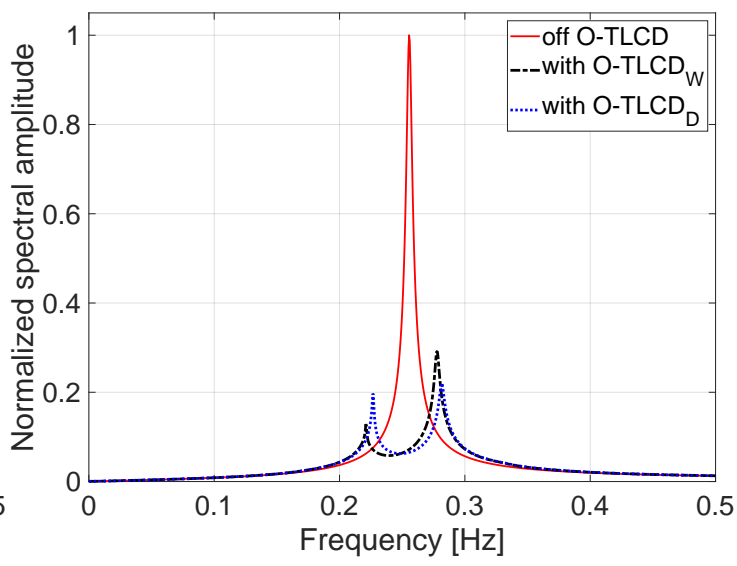

(b) $\mu=0.20$

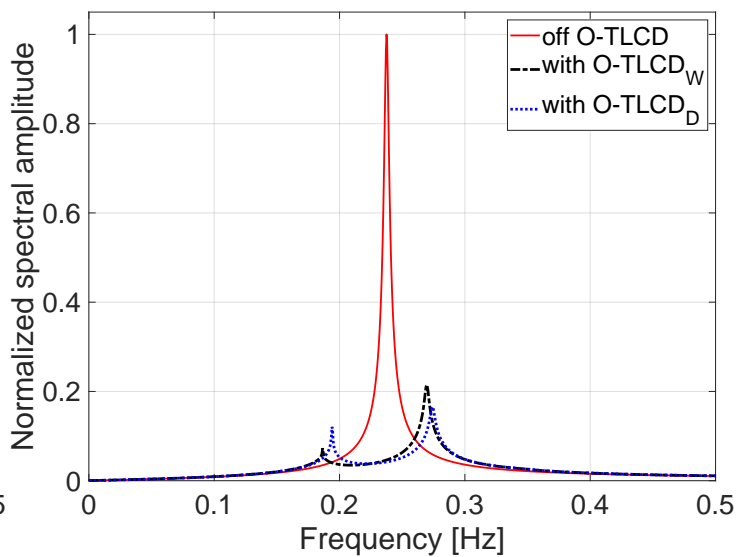

(d) $\mu=0.40$

Figure 24: Free vibration: Spectral amplitude (normalized to the peak uncontrolled amplitude) of response of the uncontrolled structure versus the controlled structure equipped with O-TLCDs with Warburton's optimal frequency $\left(\mathrm{O}_{-} \mathrm{TLCD}_{W}\right)$ and with theoretic O-TLCDs with Den Hartog's optimal frequency $\left(\mathrm{O}-\mathrm{TLCD}_{D}\right)$ when $\delta_{\text {req }}=1.310 \mathrm{~m}^{-1}$ for all mass ratios and $0.15 \mathrm{~m}$ initial displacement are applied to the structure.

In the third part of the investigation, the O-TLCD is excited harmonically by the shaking table according to Eq. 48 with $A=8.4 \mathrm{~mm}$ and the obtained resonance frequency of the O-TLCD $f=0.830 \mathrm{~Hz}$. Also, these tests are repeated for seven excitation angles $\alpha$, ranging from 0 to $90^{\circ}$ with the increment of $15^{\circ}$ with respect to the motion direction of the shaking table.

To compare with the experimental results, the O-TLCD response is simulated numerically under the same harmonic excitation for the considered $\alpha$ values. As in the first part of the investigation, the natural frequency $f_{d}$ and the head loss coefficient $\delta$ are tuned to the peak response $\left(u_{1, \max }=\right.$ $68.2 \mathrm{~mm}$ ) at $\alpha=0^{\circ}$. Accordingly, the natural frequency used in the simulations is $f_{d}=0.830 \mathrm{~Hz}$ and the head loss coefficient is $\delta=1.63 \cdot 10^{-3} \mathrm{~mm}^{-1}$. Introducing $f_{d}$ and $\delta$ into Eq. 18, the response of the pseudo TLCD $u$ is obtained. According to this equation, the response $u$ is independent from the excitation direction $\alpha$, since the assumed TLCD is defined to be always aligned with $\alpha$. On the other hand, as proposed by Eq. 1, the response of the O-TLCD is expected to change depending on $\alpha$. The liquid motion $u_{1}$ of the measured L-arm 1 is calculated by introducing the corresponding excitation direction $\alpha$, L-arm orientation $\theta=0^{\circ}$ and the calculated liquid deflection 


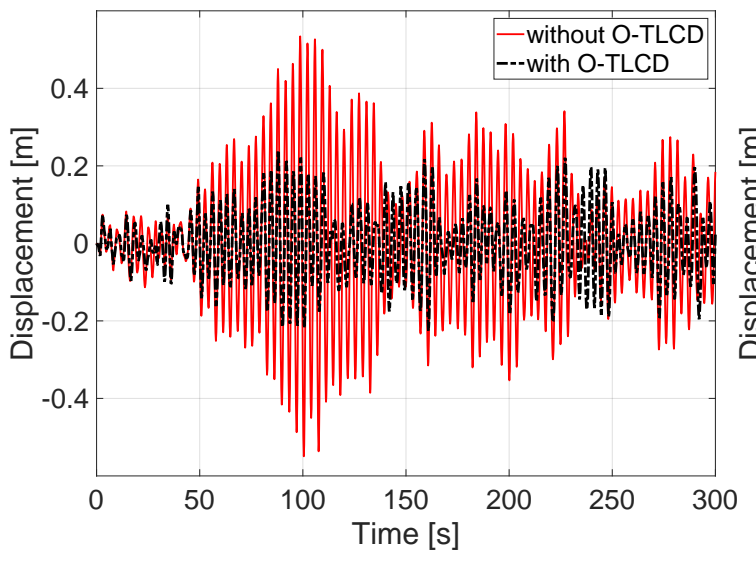

(a) $\mu=0.10$

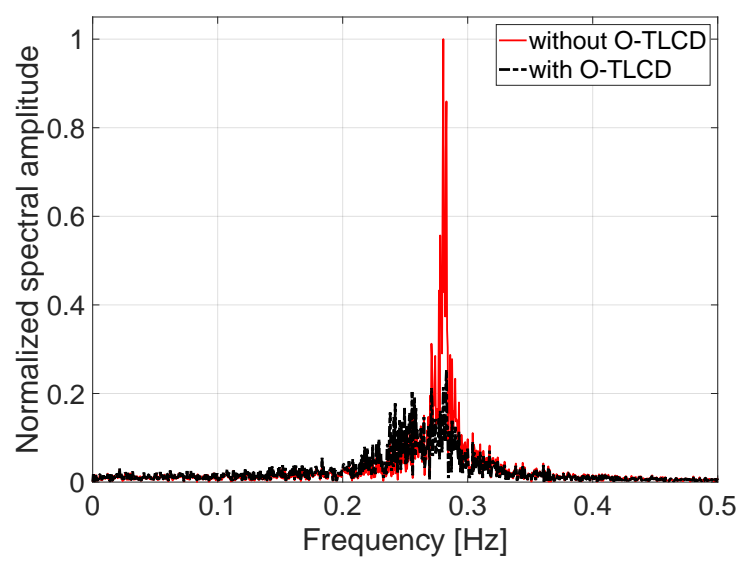

(c) $\mu=0.10$

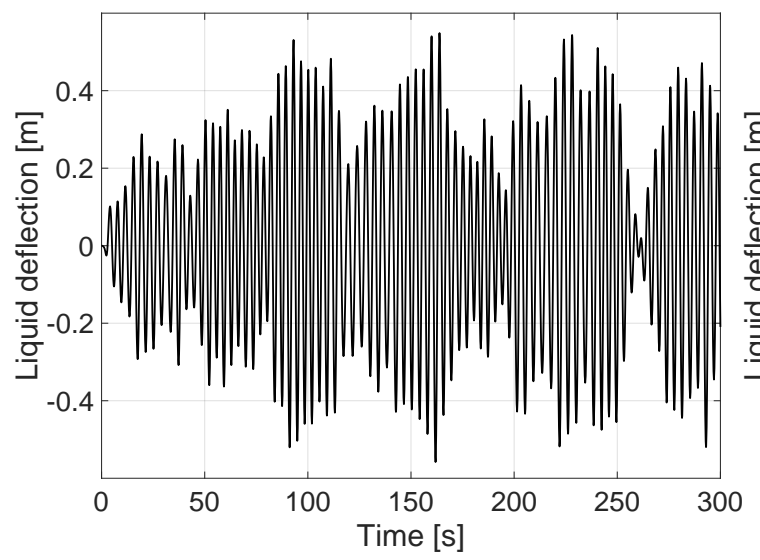

(e) $\mu=0.10$

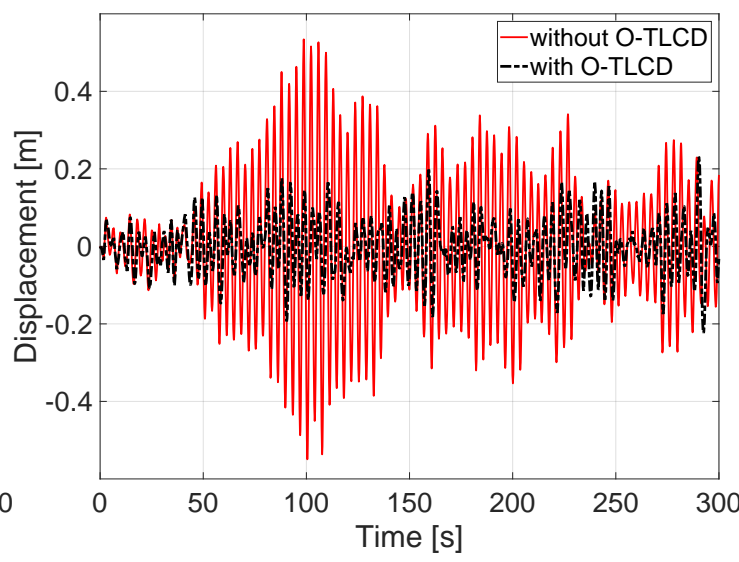

(b) $\mu=0.39$

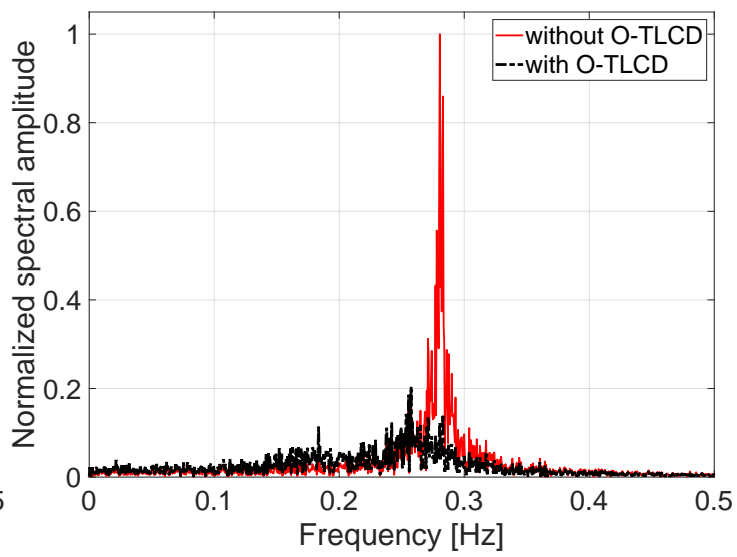

(d) $\mu=0.39$

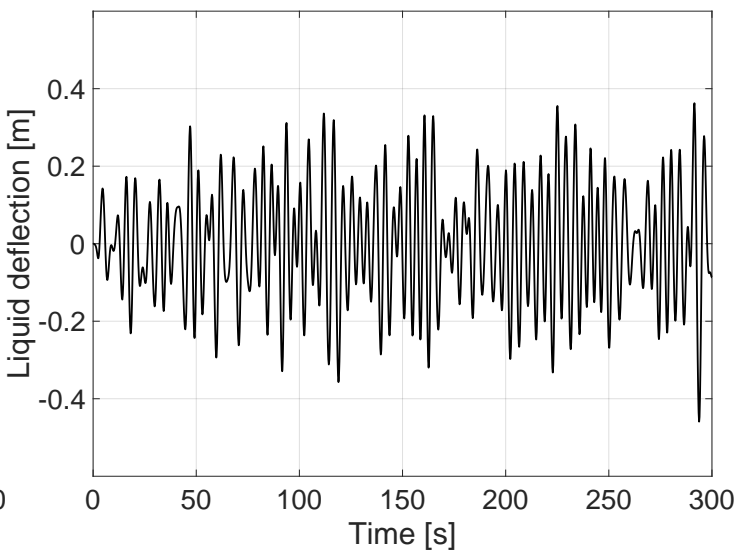

(f) $\mu=0.39$

Figure 25: White noise excitation: Displacement response of the uncontrolled structure versus the controlled structure equipped with O-TLCD ${ }_{\mu=0.10}$ with $\delta_{r e q}=0.579 \mathrm{~m}^{-1}$ and O-TLCD ${ }_{\mu=0.39}$ with $\delta_{r e q}=1.310 \mathrm{~m}^{-1}$ in the time $(\mathrm{a}, \mathrm{b})$ and the frequency domain (c,d); the corresponding liquid deflections $u$ (from Eq. 30 in (e) and (f), respectively, under white noise with sample time of $0.01 \mathrm{~s}$ and noise power of 0.005 . 


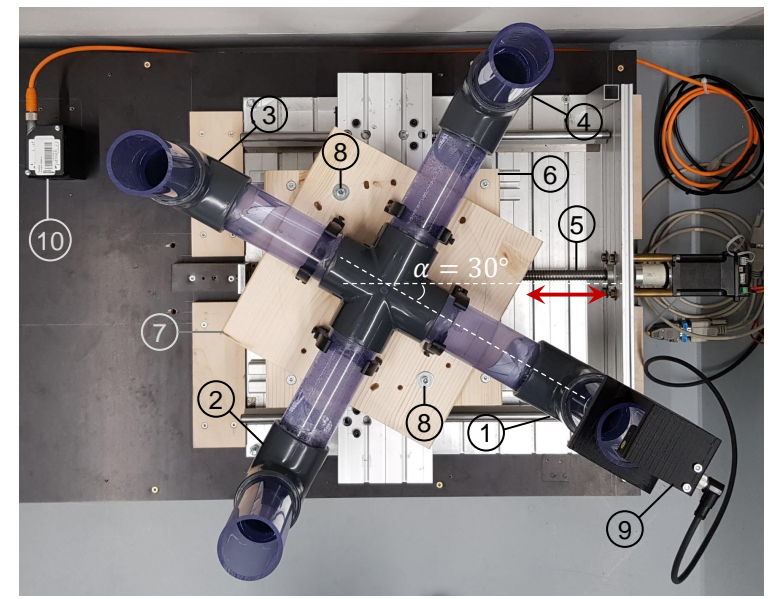

(a)

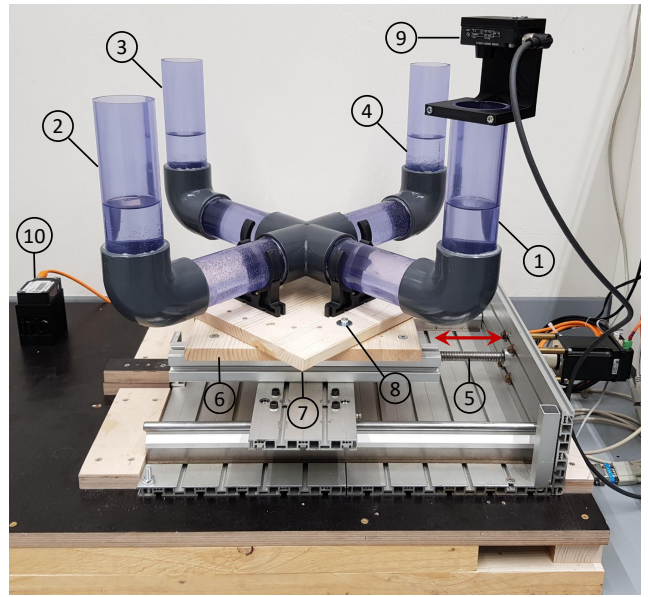

(b)

Figure 26: The fabricated prototype of the O-TLCD with four L-arms (1-4) attached on the uniaxial shaking table $(5)$ in the plan view (a) and the front view (b). A lower platform (6) is connected with a rotatable upper platform (7) at two diagonal points (8) allowing multidirectional vibration tests with the excitation direction $\alpha=0$ to $90^{\circ}$ with $15^{\circ}$ increment. Here the O-TLCD is shown at $\alpha=30^{\circ}$. The liquid deflection is measured by an ultrasonic sensor (9). The motion of the shaking table is measured by a laser sensor (10).

$u$ of the pseudo TLCD into Eq. 1.

Fig. 29 compares the time histories of the numerically calculated liquid motion $u_{1}$ with the experimental one at $\alpha=\left\{0^{\circ}, 30^{\circ}, 60^{\circ}, 90^{\circ}\right\}$. It is seen that the proposed mathematical model of the O-TLCD predicts the experimental data accurately. The liquid deflection amplitude changes depending on the excitation direction and matches the experimental data. As previously observed in the frequency spectra in Fig. 27, it is again noted here that the simulation slightly overestimates the inherent damping. Particularly in the response time history of $\alpha=60^{\circ}$, the simulated response $\left(u_{1, \max }=33.6 \mathrm{~mm}\right)$ is slightly lower than the experimental result $\left(u_{1, \max }=36.2 \mathrm{~mm}\right)$.

During experiments, by rotating the O-TLCD from $\alpha=0$ to $90^{\circ}$, we observed on the liquid surface a gradually increasing wave formation in the excitation direction. At $\alpha=90^{\circ}$, the measured L-arm is perpendicular to the excitation direction and the liquid deflection at this excitation is theoretically expected to be 0 . However, from the recorded time history shown in Fig. 29 the wave-induced liquid motion can be clearly seen. This response can be modeled by mathematical approaches proposed for TLDs and therefore does not belong to the scope of the O-TLCD mathematical model. Physically the wave formation can be prevented in O-TLCD by dividing the column vertically in smaller cells.

Fig. 30 gathers the experimental and numerical peak liquid motions for all considered excitation angles. With increasing $\alpha$, the predicted peak liquid deflection $\left(u_{1, \max }\right) u_{1}$ reduces corresponding to the experimental results. The curve belonging to the experimental results includes three measured maximum deflection values and a mean value for each excitation angle. From the comparison of the results we see particularly at $\alpha=90^{\circ}$ a marginal difference in the predicted liquid deflection due to the previously mentioned numerically overestimated inherent damping and the wave effects. Therefore, it is concluded that the proposed mathematical approach is able to imitate the behavior of the O-TLCDs markedly where the liquid motions in any liquid column can be determined for any excitation direction. 


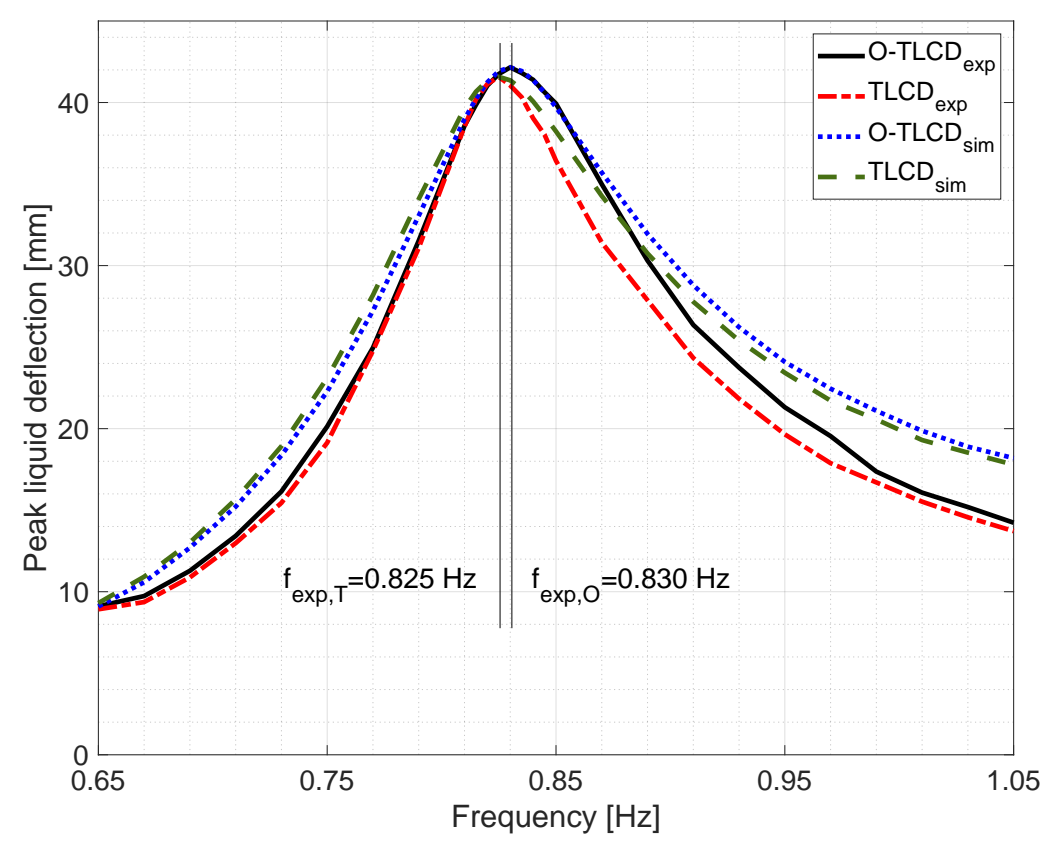

Figure 27: Experimental $\left(\mathrm{O}-\mathrm{TLCD}_{\exp }\right.$, TLCD $\left.\mathrm{D}_{\mathrm{exp}}\right)$ and numerical $\left(\mathrm{O}-\mathrm{TLCD}_{\mathrm{sim}}, \mathrm{TLCD}_{\text {sim }}\right)$ frequency response curves of the liquid deflection. The numerical calculations are performed for O-TLCD with the natural frequency $f_{d}=$ $0.830 \mathrm{~Hz}$ and the head loss coefficient $\delta=2.19 \cdot 10^{-3} \mathrm{~mm}^{-1}$. For TLCD the natural frequency is $f_{d}=0.825 \mathrm{~Hz}$ and the head loss coefficient is $\delta=2.26 \cdot 10^{-3} \mathrm{~mm}^{-1}$. Both dampers are subjected to a harmonic excitation $w=$ $4.5 \sin (2 \pi f t) \mathrm{mm}$ and are aligned with the excitation direction $\left(\alpha=0^{\circ}\right)$.

\section{Conclusion}

This paper proposed a mathematical modeling and optimization scheme for omnidirectional tuned liquid column dampers (O-TLCDs), which are formed by circularly distributed of $n$ (integer $n \geq 3$ ) L-arms about a common joint region at the center, through which the liquid moves between horizontal tubes. Accordingly, introducing the concept of a pseudo TLCD with the liquid deflection $u$ in the direction of an excitation $\alpha$, this study introduced a formal solution to determine the DoF of the O-TLCDs, by which it was proven that the O-TLCDs behave as an SDoF system; the liquid motion in each L-arm can be then obtained using $u$ and $\alpha$. Next, The paper derived the equations of motion of the O-TLCDs using Lagrange's principle and the coupled system using equilibrium of forces and showed that, owing to its configuration, O-TLCDs can reinforce structures with full counteracting force capacity in all transversal directions regardless of the excitation angle of incidence. For designing O-TLCDs, a set of design criteria and a general optimization scheme, which includes the online simulation of the system equipped with the O-TLCD exposed to an arbitrary excitation, were also proposed. Numerical parametric studies on a SDoF structure with an O-TLCD were performed for varying mass ratios and head loss coefficients. Seismic, harmonic and white noise excitations were investigated in time and frequency domains showing the high vibration reduction capability of the damper.

Aiming to verify the proposed mathematical approach, experimental investigations were per- 


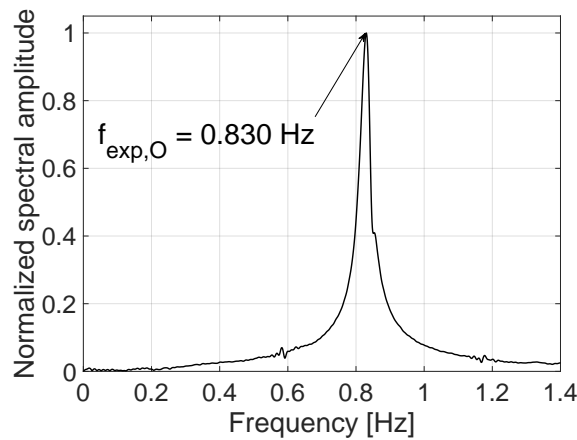

(a) $\alpha=0^{\circ}$

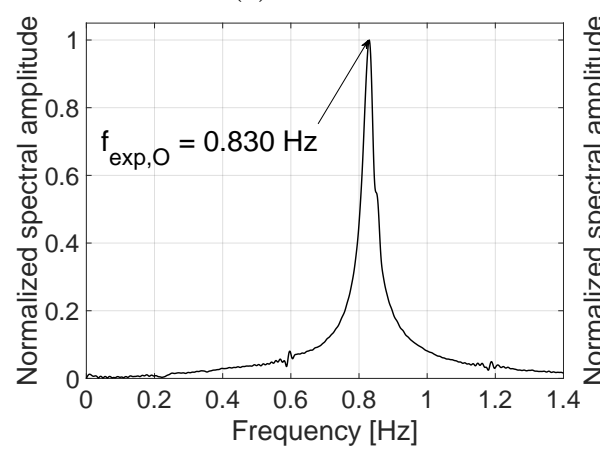

(c) $\alpha=30^{\circ}$

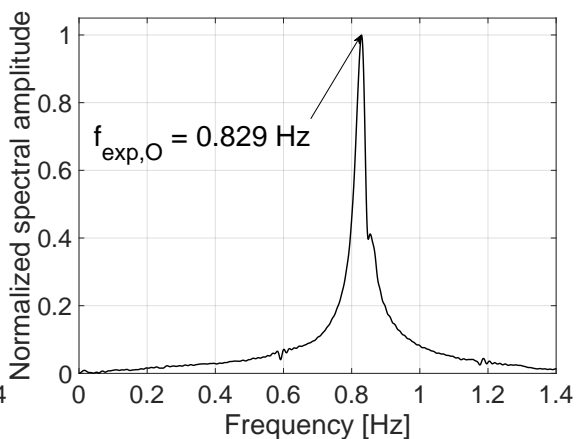

(b) $\alpha=15^{\circ}$

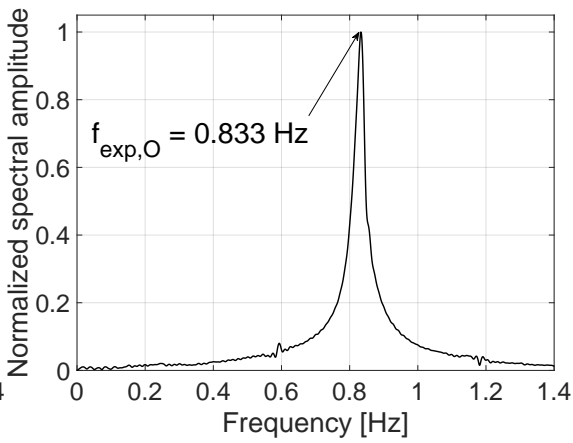

(d) $\alpha=45^{\circ}$

Figure 28: Free vibration: Spectral amplitude of the liquid deflection (normalized to the peak deflection) of the prototype O-TLCD with four orientation angles $\alpha=0$ to $\alpha=45^{\circ}$ with respect to the axis of the shaking table.

formed on an O-TLCD prototype. The prototype was attached to the shaking table and tested under harmonic vibrations, where the liquid motion was measured using an ultrasonic sensor. The natural frequency of the O-TLCD was derived from frequency sweep test. The response of the O-TLCD was measured for different excitation angles ranging from 0 to $90^{\circ}$. Observations showed that the proposed mathematical model can predict the response of O-TLCDs with circularly distributed $n$ L-arms successfully.

Results of this study introduces a mathematical description for the O-TLCDs, which requires no extra DoFs and is valid for any lateral excitation. The both numerical and experimental observations regarding the actual optimal frequency and the required head loss coefficient for a vibration scenario highlights also the need for a semi-active tuning capacity for the introduced O-TLCDs, which necessitates further investigations.

\section{Acknowledgment}

The authors would like to express their sincere appreciation for the financial support from the German Federal Ministry of Education and Research (Bundesministerium für Bildung und Forschung, BMBF) with the grant number: FKZ 03VP04680. The authors would also like to thank the Control Engineering Institute of RWTH Aachen University, and in particular Mr. Markus Zimmer, for their support during the preparation of the experimental setup. 


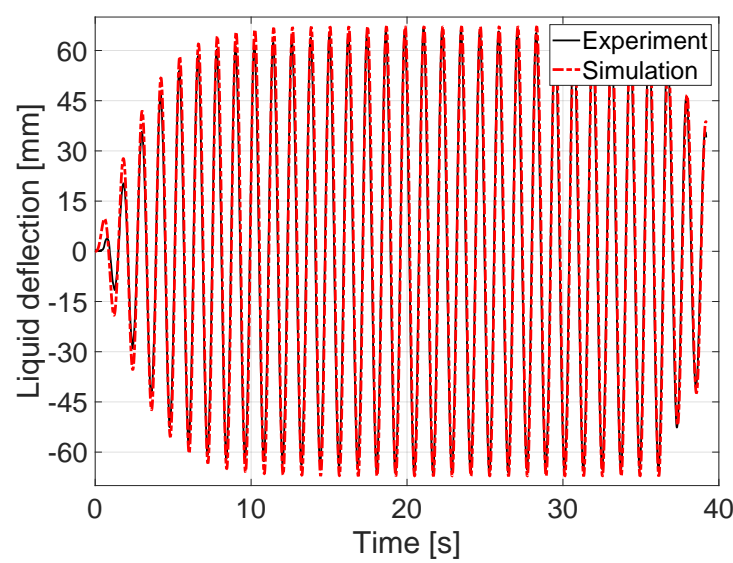

(a) $\alpha=0^{\circ}$

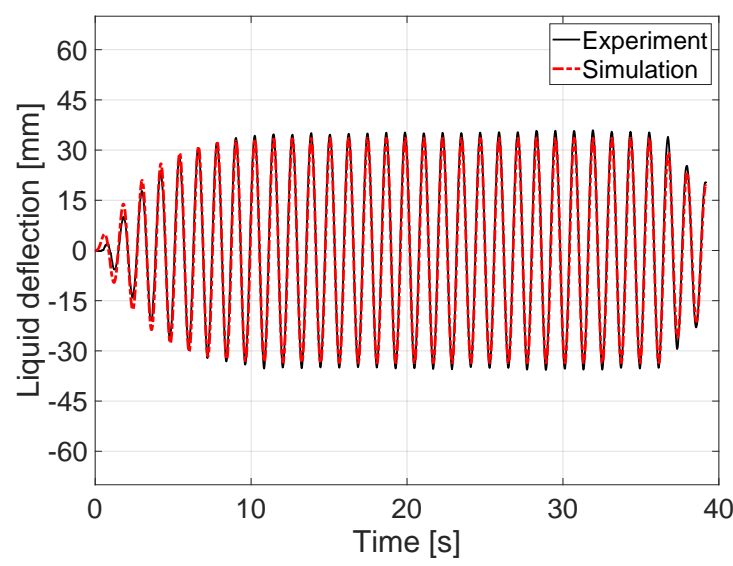

(c) $\alpha=60^{\circ}$

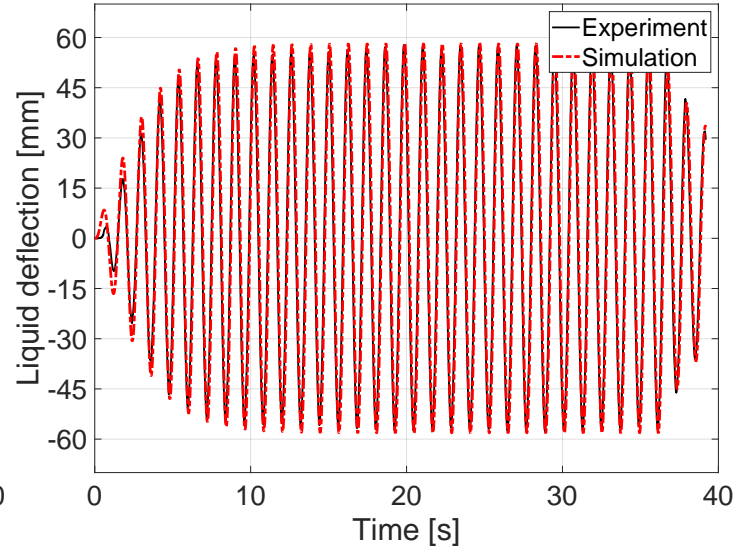

(b) $\alpha=30^{\circ}$

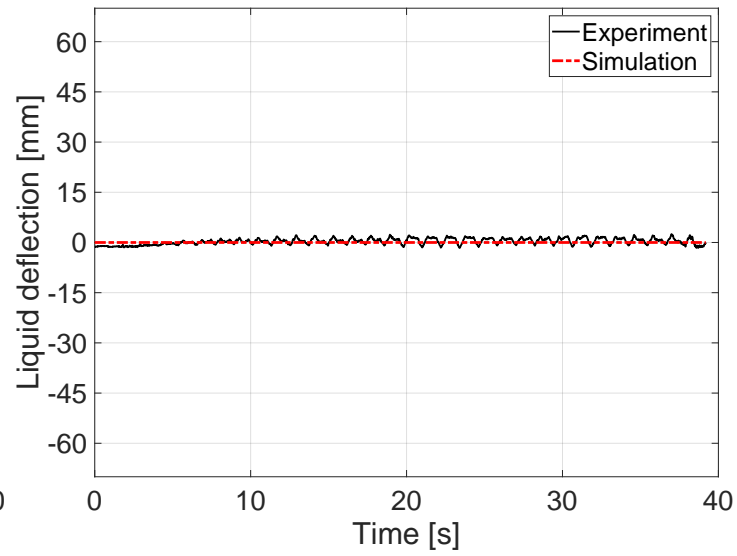

(d) $\alpha=90^{\circ}$

Figure 29: Comparison of the experimental and numerical liquid deflection $u_{1}$ of the O-TLCD subjected to a harmonic excitation $w=8.4 \sin (2 \pi 0.83 t) \mathrm{mm}$ at the excitation direction $\alpha=\left\{0^{\circ}, 30^{\circ}, 60^{\circ}, 90^{\circ}\right\}$. At $\alpha=0^{\circ}$, the observed Larm 1 of the O-TLCD is aligned with the excitation direction. At $\alpha=90^{\circ}$, the observed L-arm of the O-TLCD is perpendicular to the excitation direction. The natural frequency is $f_{d}=0.830 \mathrm{~Hz}$ and the head loss coefficient is $\delta=1.63 \cdot 10^{-3} \mathrm{~mm}^{-1}$. 


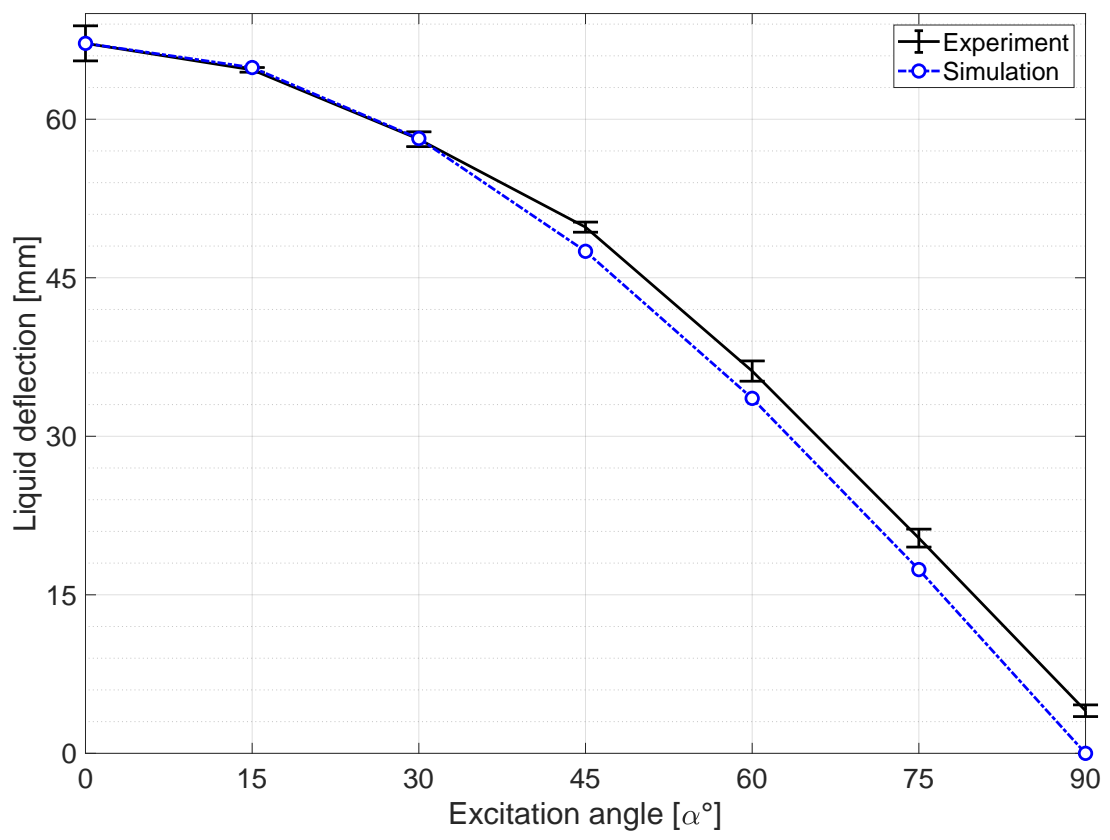

Figure 30: Comparison of the experimental and numerical peak liquid deflection $\left(u_{1, \max }\right) u_{1}$ of the O-TLCD subjected to a harmonic excitation $w=8.4 \sin (2 \pi 0.83 t) \mathrm{mm}$ at the excitation direction $\alpha=0$ to $90^{\circ}$. At $\alpha=0^{\circ}$, the observed L-arm 1 of the O-TLCD is aligned with the excitation direction. At $\alpha=90^{\circ}$, the observed L-arm 1 of the O-TLCD is perpendicular to the excitation direction. The natural frequency is $f_{d}=0.830 \mathrm{~Hz}$ and the head loss coefficient is $\delta=1.63 \cdot 10^{-3} \mathrm{~mm}^{-1}$. 


\section{References}

[1] J. T. P. Yao, Concept of structural control, ASCE Journal of Structural Division 98 (1972) 1567-1574.

[2] G. Housner, L. Bergman, T. Caughey, A. Chassiakos, R. Claus, S. Masri, E. Skelton, T. Soong, B. Spencer, J. Yao, Structural control: past, present and future, Journal of Engineering Mechanics 123 (9) (1997) 897-974.

[3] O. El-Khoury, H. Adeli, Recent advances on vibration control of structures under dynamic loading, Archives of Computational Methods in Engineering 20 (4) (2013) 353-360.

[4] T. T. Soong, Active structural control: theory and practice, Longman Wiley, London, England, 1990.

[5] T. T. Soong, G. F. Dargush, Passive energy dissipation systems in structural engineering, John Wiley \& Sons, Inc., New York, 1997.

[6] M. D. Symans, M. C. Constantinou, Semi-active control systems for seismic protection of structures: a stateof-the-art review, Engineering Structures 21 (6) (1999) 469-487.

[7] B. Mehrkian, A. Bahar, A. Chaibakhsh, Semiactive conceptual fuzzy control of magnetorheological dampers in an irregular base-isolated benchmark building optimized by multi-objective genetic algorithm, Structural Control and Health Monitoring 26 (3) (2019) e2302.

[8] H. Frahm, Means for damping the rolling motion of ships, US-Patent 970368. (1910).

[9] F. Sakai, S. Takaeda, Tuned liquid column damper - new type device for suppression of building vibrations, in: Proceedings of International Conference on High Rise Buildings, 1989, p. 926-931.

[10] H. Gao, K. Kwok, B. Samali, Optimization of tuned liquid column dampers, Engineering Structures 19 (6) (1997) 476-486.

[11] P. Hitchcock, K. Kwok, R. Watkins, Characteristics of liquid column vibration absorbers (LCVA)-i, Engineering Structures 19 (2) (1997) 126-134.

[12] S. D. Xue, J. M. Ko, Y. L. Xu, Optimum parameters of tuned liquid column damper for suppressing pitching vibration of an undamped structure, Journal of Sound and Vibration 235 (4) (2000) 639-653.

[13] S. L. T. de Souza, I. L. Caldas, R. L. Viana, J. M. Balthazar, R. M. L. R. F. Brasil, Dynamics of vibrating systems with tuned liquid column dampers and limited power supply, Journal of Sound and Vibration 289 (4) (2006) 987-998.

[14] J.-C. Wu, M.-H. Shih, Y.-Y. Lin, Y.-C. Shen, Design guidelines for tuned liquid column damper for structures responding to wind, Engineering Structures 27 (13) (2005) 1893-1905.

[15] K. Shum, Closed form optimal solution of a tuned liquid column damper for suppressing harmonic vibration of structures, Engineering Structures 31 (2009) 84-92.

[16] J.-C. Wu, C.-H. Chang, Y.-Y. Lin, Optimal designs for non-uniform tuned liquid column dampers in horizontal motion, Journal of Sound and Vibration 326 (1) (2009) 104-122.

[17] S. Chakraborty, R. Debbarma, G. C. Marano, Performance of tuned liquid column dampers considering maximum liquid motion in seismic vibration control of structures, Journal of Sound and Vibration 331 (7) (2012) 1519-1531.

[18] S.-K. Lee, H.-R. Lee, K.-W. Min, Experimental verification on nonlinear dynamic characteristic of a tuned liquid column damper subjected to various excitation amplitudes, The Structural Design of Tall and Special Buildings 21 (5) (2012) 374-388.

[19] S. Bhattacharyya, A. Ghosh, B. Basu, Nonlinear modeling and validation of air spring effects in a sealed tuned liquid column damper for structural control, Journal of Sound and Vibration 410 (2017) 269-286.

[20] C. Chang, C. Hsu, Control performance of liquid column vibration absorbers, Engineering Structures 20 (7) (1998) 580-586.

[21] T. Balendra, C. Wang, G. Rakesh, Vibration control of various types of buildings using TLCD, Journal of Wind Engineering and Industrial Aerodynamics 83 (1999) 197-208.

[22] S. Yalla, A. Kareem, Optimum absorber parameters for tuned liquid column dampers, Journal of Structural Engineering ASCE 126 (8) (2000) 906-915.

[23] A. Ghosh, B. Basu, Seismic vibration control of short period structures using the liquid column damper, Engineering Structures 26 (13) (2004) 1905-1913.

[24] J. L. P. Felix, J. M. Balthazar, R. M. L. R. F. Brasil, On tuned liquid column dampers mounted on a structural frame under a non-ideal excitation, Journal of Sound and Vibration 282 (3) (2005) 1285-1292.

[25] M. Reiterer, F. Ziegler, Control of pedestrian-induced vibrations of long-span bridges, Structural Control and Health Monitoring 13 (2006) 1003-1027.

[26] K. Al-Saif, K. Aldakkan, M. Foda, Modified liquid column damper for vibration control of structures, International Journal of Mechanical Sciences 53 (2011) 505-512.

[27] Y. Bigdeli, D. Kim, Damping effects of the passive control devices on structural vibration control: TMD, TLC and TLCD for varying total masses, KSCE Journal of Civil Engineering 20 (1) (2016) 301-308. 
[28] Y. Yu, L. Xu, L. Zhang, Experimental study on variation rules of damping with influential factors of tuned liquid column damper, Shock and Vibration 2017 (Article ID 6209205) (2017) 17 pages.

[29] A. Di Matteo, T. Furtmüller, C. Adam, A. Pirrotta, Optimal design of tuned liquid column dampers for seismic response control of base-isolated structures, Acta Mechanica 229 (2) (2018) 437-454.

[30] A. C. Altunisik, A. Yetisken, V. Kahya, Experimental study on control performance of tuned liquid column dampers considering different excitation directions, Mechanical Systems and Signal Processing 102 (2018) 59-71.

[31] T. Furtmüller, A. Di Matteo, C. Adam, A. Pirrotta, Base-isolated structure equipped with tuned liquid column damper: An experimental study, Mechanical Systems and Signal Processing 116 (2019) 816-831.

[32] S. Yalla, A. Kareem, Semiactive tuned liquid column dampers: Experimental study, Journal of Structural Engineering 129 (2003) 960-971.

[33] Y.-H. Chen, C.-H. Ko, Active tuned liquid column damper with propellers, Earthquake Engineering \& Structural Dynamics 32 (10) (2003) 1627-1638.

[34] Y. Q. Ni, Z. G. Ying, J. Y. Wang, J. M. Ko, B. F. Spencer, Stochastic optimal control of wind-excited tall buildings using semi-active MR-TLCDs, Probabilistic Engineering Mechanics 19 (3) (2004) 269-277.

[35] M. J. Hochrainer, F. Ziegler, Control of tall building vibrations by sealed tuned liquid column dampers, Structural Control and Health Monitoring 13 (6) (2006) 980-1002.

[36] N. Luo, C. L. Bottasso, H. R. Karimi, M. Zapateiro, Semiactive control for floating offshore wind turbines subject to aero-hydrodynamic loads, in: Proceedings of the International Conference on Renewable Energies and Power Quality, 2011.

[37] E. Sonmez, S. Nagarajaiah, C. Sun, B. Basu, A study on semi-active tuned liquid column dampers (sTLCDs) for structural response reduction under random excitations, Journal of Sound and Vibration 362 (3) (2016) 1-15.

[38] V. D. La, C. Adam, General on-off damping controller for semi-active tuned liquid column damper, Journal of Vibration and Control (2016) 1-15.

[39] M. J. Hochrainer, P. A. Fotiu, Design of coupled tuned liquid column gas dampers for multi-mode reduction in vibrating structures, Acta Mechanica 229 (2) (2018) 911-928.

[40] O. Altay, S. Klinkel, A semi-active tuned liquid column damper for lateral vibration control of high-rise structures: Theory and experimental verification, Structural Control and Health Monitoring 25 (12) (2018) e2270.

[41] S. Sarkar, A. Chakraborty, Development of semi-active vibration control strategy for horizontal axis wind turbine tower using multiple magneto-rheological tuned liquid column dampers, Journal of Sound and Vibration 457 (2019) 15-36.

[42] M. Hochrainer, C. Adam, F. Ziegler, Application of tuned liquid column dampers for passive structural control, in: Proceeding of 7th International Congress on Sound and Vibration (ICSV 7), Vol. CD-ROM paper, paper no 393, 2000, pp. 3107-3114.

[43] C. Fu, Application of torsional tuned liquid column gas damper for plan-asymmetric buildings, Structural Control and Health Monitoring 18 (5) (2011) 492-509.

[44] L. Feipeng, L. Ruyi, B. Haifeng, X. Mingming, X. Jiayun, Research on wind-induced torsional control with annular damper for high-rise building, in: 2016 International Conference on Smart City and Systems Engineering (ICSCSE), 2016, pp. 439-444.

[45] B. Basu, Z. Zhang, S. R. K. Nielsen, Damping of edgewise vibration in wind turbine blades by means of circular liquid dampers, Wind Energy 19 (2) (2016) 213-226.

[46] J.-S. Heo, S.-K. Lee, E. Park, S.-H. Lee, K.-W. Min, H. Kim, J. Jo, B.-H. Cho, Performance test of a tuned liquid mass damper for reducing bidirectional responses of building structures, The Structural Design of Tall and Special Buildings 18 (7) (2009) 789-805.

[47] K.-W. Min, J. Kim, H.-R. Lee, A design procedure of two-way liquid dampers for attenuation of wind-induced responses of tall buildings, Journal of Wind Engineering and Industrial Aerodynamics 129 (2014) 22-30.

[48] S.-K. Lee, K.-W. Min, H.-R. Lee, Parameter identification of new bidirectional tuned liquid column and sloshing dampers, Journal of Sound and Vibration 330 (7) (2011) 1312-1327.

[49] F. Sakai, T. T. Takaeda, Damping device for tower-like structure, US-Patent 5070663 (1991).

[50] X. T. Zhang, R. C. Zhang, Y. L. Xu, Analysis on control of flow-induced vibration by tuned liquid damper with crossed tube-like containers, Journal of Wind Engineering and Industrial Aerodynamics 50 (1993) 351-360.

[51] P. Hitchcock, K. Kwok, R. Watkins, Characteristics of liquid column vibration absorbers (LCVA)-ii, Engineering Structures 19 (2) (1997) 135-144.

[52] L. Rozas, R. L. Boroschek, A. Tamburrino, M. Rojas, A bidirectional tuned liquid column damper for reducing the seismic response of buildings, Structural Control and Health Monitoring 23 (4) (2016) 621-640.

[53] X. Tong, X. Zhao, A. Karcanias, Passive vibration control of an offshore floating hydrostatic wind turbine model, Wind Energy 21 (9) (2018) 697-714. 
[54] C. Coudurier, O. Lepreux, N. Petit, Modelling of a tuned liquid multi-column damper. Application to floating wind turbine for improved robustness against wave incidence, Ocean Engineering 165 (2018) 277-292.

[55] Y. A. Cengel, J. M. Cimbala, Fluid Mechanics: Fundamentals and Applications, 4th Edition in SI Units, McGraw-Hill Education, New York, NY, 2018.

[56] M. J. Hochrainer, Control of vibrations of civil engineering structures with special emphasis on tall buildings, Doctoral dissertation, Vienna University of Technology, Austria (2001).

[57] G. B. Warburton, Optimum absorber parameters for various combinations of response and excitation parameters, Earthquake Engineering \& Structural Dynamics 10 (3) (1982) 381-401.

[58] J. P. Den Hartog, Mechanical vibrations, McGraw-Hill, New York, 1947.

[59] F. M. White, Fluid Mechanics, 8th Edition, McGraw-Hill Education, New York, United States, 2016. 